Article

\title{
Stepwise Introduction of Different Substituents to $\alpha$-Chloro- $\omega$-hydrooligosilanes: Convenient Synthesis of Unsymmetrically Substituted Oligosilanes
}

\author{
Ken-ichiro Kanno *, Yumi Aikawa, Yuka Niwayama, Misaki Ino, \\ Kento Kawamura and Soichiro Kyushin *D \\ Division of Molecular Science, Graduate School of Science and Technology, Gunma University, \\ Kiryu, Gunma 376-8515, Japan; yumi.a174.4@gmail.com (Y.A.); yu.niwa06@gmail.com (Y.N.); \\ misaki.ino512@gmail.com (M.I.); t12301059@gunma-u.ac.jp (K.K.) \\ * Correspondence: kkanno@gunma-u.ac.jp (K.-i.K.); kyushin@gunma-u.ac.jp (S.K.); \\ Tel.: +81-277-30-1292 (K.-i.K. \& S.K.)
}

Received: 12 August 2018; Accepted: 14 September 2018; Published: 18 September 2018

\begin{abstract}
A series of unsymmetrically substituted oligosilanes were synthesized via stepwise introduction of different substituents to $\alpha$-chloro- $\omega$-hydrooligosilanes. The reactions of $\alpha$-chloro- $\omega$ hydrooligosilanes with organolithium or Grignard reagents gave hydrooligosilanes having various alkyl, alkenyl, alkynyl and aryl groups. Thus-obtained hydrooligosilanes were converted into alkoxyoligosilanes by ruthenium-catalyzed dehydrogenative alkoxylation with alcohols.
\end{abstract}

Keywords: $\alpha$-chloro- $\omega$-hydrooligosilane; titanium; ruthenium; dehydrogenative alkoxylation

\section{Introduction}

Recently, various functionalized oligosilanes have attracted growing interests due to their unique photophysical and electronic properties [1-13]. In the synthesis of such compounds, introduction of functionality to oligosilanes is an important process. Oligosilanes having both chlorosilane and hydrosilane moieties are especially interesting because two different functionalities could be introduced to the oligosilanes. For example, $\alpha$-chloro- $\omega$-hydrooligosilanes are potential precursors for such modification.

A problem to be overcome is limitation of facile functionalization of the hydrosilane moiety in the oligosilanes. Thus far, halogenation [14-16], Lewis acid-catalyzed reactions [17-19] and radical-promoted reactions [20-24] have been used for the $\mathrm{Si}-\mathrm{H}$ modification of hydrooligosilanes. Transition metal-catalyzed reactions of hydrosilanes are well known as $\mathrm{Si}-\mathrm{H}$ conversion methods, including hydrosilylation [25-27], dehydrogenative silylation of $\mathrm{OH}$ and $\mathrm{NH}$ groups [28-31], silylation of aromatic rings [32-36] and so on. however, most of them are applicable only to hydromonosilanes. When hydrooligosilanes are subjected to these transition metal-catalyzed reactions, major products come from the cleavage of $\mathrm{Si}-\mathrm{Si}$ bonds. Although the transition metal-catalyzed reactions are utilized as valuable organic synthesis methodologies [37-40], it is not the case for the transformation of hydrooligosilanes. As a rare example, Yamanoi and Nishihara have reported the palladium-catalyzed arylation of hydrooligosilanes with aryl iodides to afford the corresponding arylated oligosilanes with preservation of the $\mathrm{Si}-\mathrm{Si}$ bonds [41,42].

Previously, we reported titanium-catalyzed synthesis of the hydrogen-terminated oligosilanes from $\alpha, \omega$-dichlorooligosilanes [43]. This method enabled us to synthesize $\alpha$-chloro- $\omega$-hydrooligosilanes with high selectivity. The $\alpha$-chloro- $\omega$-hydrooligosilanes are good precursors of variously substituted hydrooligosilanes via nucleophilic substitution of the chlorosilane moiety. Furthermore, we have recently found ruthenium-catalyzed dehydrogenative alkoxylation of a hydrodisilane 
with alcohols [44]. Interestingly, the reactions proceed with preserving the $\mathrm{Si}-\mathrm{Si}$ bond despite its susceptible nature to transition metals [37-40]. Combination of these two methods leads to convenient synthesis of unsymmetrically substituted oligosilanes. In this paper, we report the synthesis of various substituted hydrooligosilanes from $\alpha$-chloro- $\omega$-hydrooligosilanes and transformation of thus-obtained hydrooligosilanes to the corresponding alkoxy oligosilanes.

\section{Results and Discussion}

\subsection{Synthesis of 1-Hydrooligosilanes}

As reported previously, $\alpha$-chloro- $\omega$-hydrooligosilanes $1-3$ are synthesized by the selective monoreduction of $\alpha, \omega$-dichlorooligosilanes with alkylmagnesium chlorides in the presence of a catalytic amount of $\mathrm{TiCl}_{4}$ (Scheme 1) [43]. The remaining chlorosilane moiety is possible functionality for introduction of various substituents.

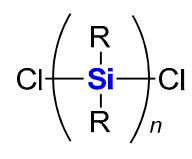

$n=2-4$

$\mathrm{R}=\mathrm{Me}, i-\mathrm{Pr}$
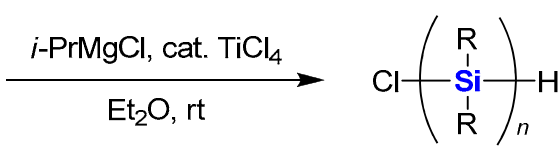

1: $\mathrm{R}=i-\mathrm{Pr}, n=2$

2: $\mathrm{R}=\mathrm{Me}, n=3$

3: $\mathrm{R}=\mathrm{Me}, n=4$

Scheme 1. Monoreduction of $\alpha, \omega$-dichlorooligosilanes with $i$-PrMgCl in the presence of a catalytic amount of $\mathrm{TiCl}_{4}$.

As shown in Scheme 2, the chlorosilane moiety of 1 was smoothly substituted by organolithium reagents. When 1 was treated with 2-thienyllithium, 2-thienyldisilane 4 was obtained in high yield. 4-Methoxyphenyl group was also introduced to afford 4-methoxyphenyldisilane 5.
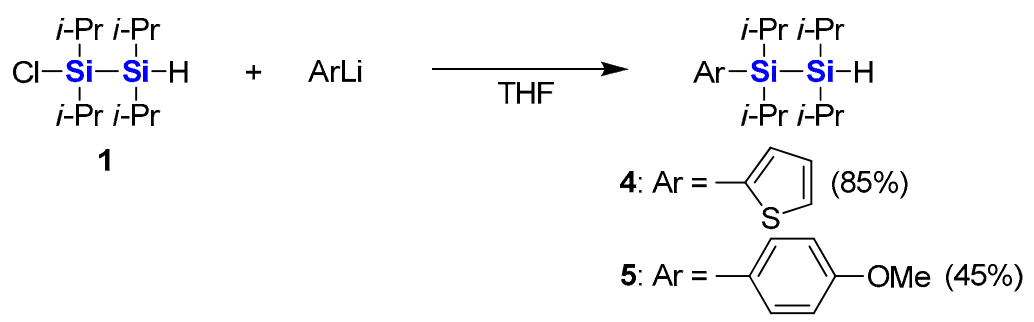

Scheme 2. Synthesis of substituted hydrodisilanes from 1-chloro-2-hydrodisilane.

In the cases of polymethylated oligosilanes 2 and 3, Grignard reagents are more suitable for the substitution of the chlorosilane moiety. The Grignard reagents were prepared in the presence of lithium chloride and DIBAL-H to activate magnesium metal [45]. As shown in Scheme 3, various alkyl, alkenyl, alkynyl and aryl groups were introduced, and substituted hydrooligosilanes were successfully synthesized. As alkyl group installation, 5-hexenyl- and 2-phenylethylmagnesium reagents were used to afford hydrotrisilanes 6 and 7. Mono- and disubstituted alkenyl groups such as styryl and 2-buten-2-yl ones were also introduced to the trisilane skeleton successfully. The stereochemistry of the alkene moieties of trisilanes 8 and 9 was determined by ${ }^{1} \mathrm{H}$ NMR spectroscopy. The $E$ geometry of the styryl group in 8 was confirmed by the $J$ value of the vinylic coupling $(19 \mathrm{~Hz})$. The major $Z$ isomer of 9 was confirmed by NOE experiment. Three types of alkynyl Grignard reagents with aliphatic, aryl and silyl substituents were subjected to the reaction to afford alkynyltrisilanes 10-12. Various aryl groups were also introduced to 2 to afford phenyl-, 4-methoxyphenyl-, 4-dimethylaminophenyl-, and 2-thienyltrisilanes 13-16. The similar arylation was also applicable to 3, and 2-thienyltetrasilane 17 was obtained. All of these reactions proceed without loss of the hydrosilane moieties, which can be used for further modification of the hydrooligosilanes. The reason for low yields in some cases is attributed to loss during the isolation procedure by column chromatography over silica gel. 


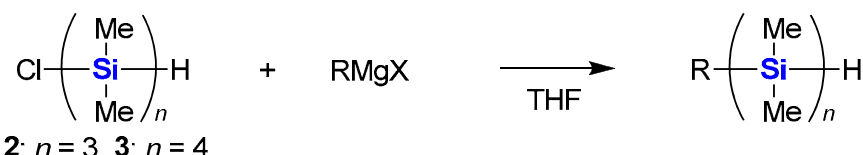

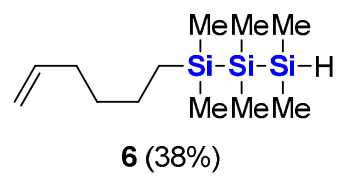

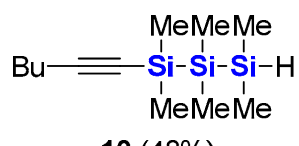

$10(48 \%)$
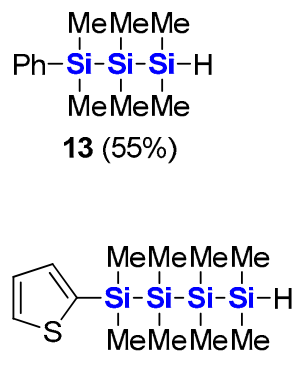

$17(43 \%)$
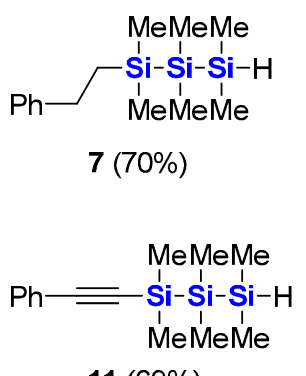

$11(69 \%)$

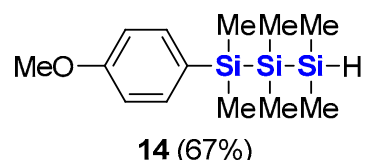

$14(67 \%)$
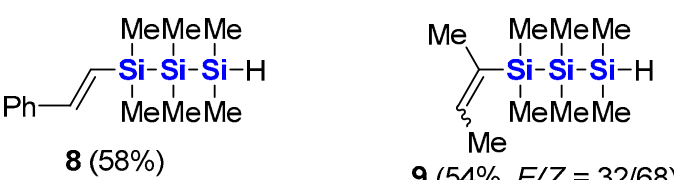

$9(54 \%, E / Z=32 / 68)$
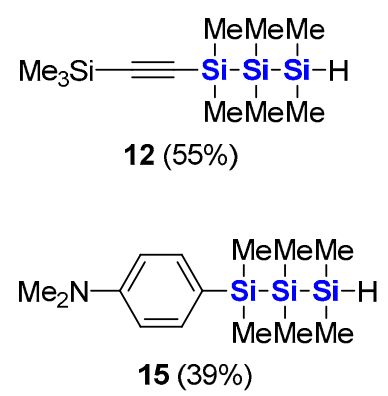

Scheme 3. Synthesis of substituted hydrooligosilanes from $\alpha$-chloro- $\omega$-hydrooligosilanes.

\subsection{Dehydrogenative Alkoxylation of hydrooligosilanes with Alcohols}

As mentioned above, we have reported ruthenium-catalyzed dehydrogenative alkoxylation of a hydrodisilane with alcohols without Si-Si bond cleavage [44]. The reactions are also applicable to various hydrotrisilanes. As shown in Scheme 4, some of the hydrotrisilanes synthesized above were subjected to the ruthenium-catalyzed dehydrogenative alkoxylation with methanol. All reactions proceeded smoothly in toluene at room temperature in the presence of $2.5 \mathrm{~mol} \%$ of $\left[\mathrm{RuCl}_{2}(p-c y m e n e)\right]_{2}$ to afford methoxytrisilanes 18-21 in good yields. It is worth noting that the alkenyl and alkynyl moieties tolerate the reactions. Neither hydrosilylation nor bis-silylation occurred under these reaction conditions.

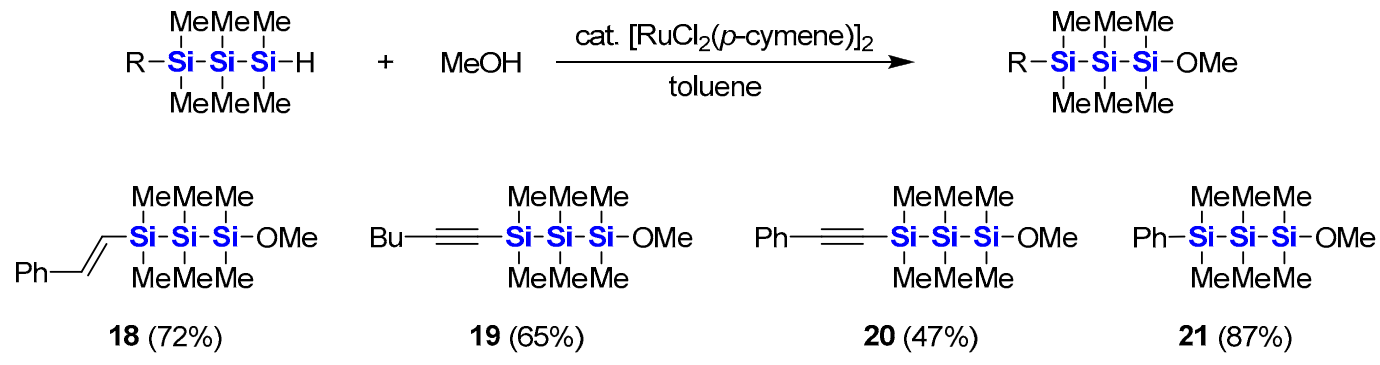

Scheme 4. Synthesis of 1-methoxytrisilanes from 1-hydrotrisilanes.

To gain further insight into the substituent effects in the ruthenium-catalyzed alkoxylation, ethyl-substituted hydrodisilane $\mathbf{2 2}$ and 2-hydrotrisilane $\mathbf{2 6}$ were used for the reactions with methanol. The results are summarized in Tables 1 and 2. Even though an excess amount of methanol (10 equiv) was used in the reaction of 22, the reaction rate is much slower than that of $\mathrm{PhMe}_{2} \mathrm{SiSiMe}_{2} \mathrm{H}$, which finished within $2 \mathrm{~h}$ under the same reaction conditions [44]. When bulkier alcohols are used, 
the reaction rate becomes slower. The reaction of 22 with ethanol needed heating at $50{ }^{\circ} \mathrm{C}$ to be completed within one day. The reaction with a large excess of 2-propanol is much more sluggish. Even though the reaction was carried out on heating, more than $40 \mathrm{~h}$ were needed for complete consumption of 22 .

Table 1. Reactions of 22 with various alcohols in the presence of $\left[\mathrm{RuCl}_{2}(p \text {-cymene) }]_{2}\right.$.

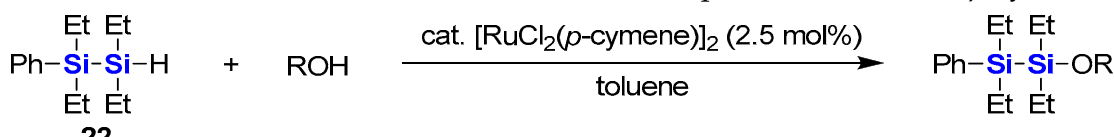

22

23: $\mathrm{R}=\mathrm{Me}$

24: $\mathrm{R}=\mathrm{Et}$

25: $\mathrm{R}=i-\mathrm{Pr}$

\begin{tabular}{cccccc}
\hline Entry & Alcohol (equiv) & Temperature & Reaction Time/h & Product & Isolated Yield/\% \\
\hline 1 & $\mathrm{MeOH}(10)$ & $0{ }^{\circ} \mathrm{C}-\mathrm{rt}$ & 20 & $\mathbf{2 3}$ & 83 \\
2 & $\mathrm{EtOH}(10)$ & $50{ }^{\circ} \mathrm{C}$ & 23 & $\mathbf{2 4}$ & 45 \\
3 & $i-\operatorname{PrOH}(100)$ & $0-50{ }^{\circ} \mathrm{C}$ & 41 & $\mathbf{2 5}$ & 58 \\
\hline
\end{tabular}

Table 2. Reactions of $\mathbf{2 6}$ with methanol in the presence of various ruthenium catalysts.

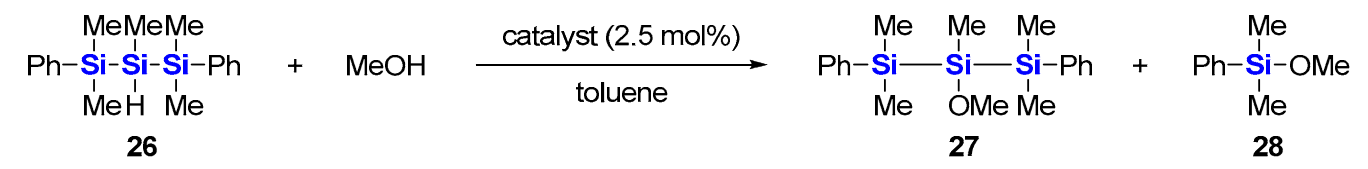

\begin{tabular}{|c|c|c|c|c|c|c|c|}
\hline \multirow{2}{*}{ Entry } & \multirow{2}{*}{ Catalyst } & \multirow{2}{*}{ Methanol (equiv) } & \multirow{2}{*}{ Temperature $/{ }^{\circ} \mathrm{C}$} & \multirow{2}{*}{ Reaction Time/h } & \multicolumn{3}{|c|}{ GC Yield $/ \%^{1}$} \\
\hline & & & & & 26 & 27 & 28 \\
\hline 1 & {$\left[\mathrm{RuCl}_{2}(p \text {-cymene })\right]_{2}$} & 10 & 0 & 23 & 19 & 16 & 47 \\
\hline 2 & {$\left[\mathrm{RuCl}_{2} \text { (mesitylene) }\right]_{2}$} & 10 & $0-50$ & 51 & 12 & 22 & 11 \\
\hline 3 & {$\left[\mathrm{RuCl}_{2} \text { (benzene) }\right]_{2}$} & 10 & $0-50$ & 100 & 3 & $59(29)$ & 5 \\
\hline 4 & $\mathrm{RuHCl}(\mathrm{CO})\left(\mathrm{PPh}_{3}\right)_{3} 2$ & 10 & 0 & 19 & 41 & 0 & 2 \\
\hline
\end{tabular}

${ }^{1}$ Isolated yield was given in parentheses. ${ }^{2} 5 \mathrm{~mol} \%$.

For the alkoxylation of 26 , optimization of the ruthenium catalyst was necessary, as shown in Table 2. The reaction of $\mathbf{2 6}$ with methanol in the presence of the ( $p$-cymene)ruthenium catalyst gave the desired 2-methoxytrisilane 27 in low yield along with monosilane 28, which was produced by $\mathrm{Si}-\mathrm{Si}$ bond cleavage (Entry 1). Changing the aromatic ligand to mesitylene slightly improved the formation of 27, but the Si-Si bond cleavage still occurred significantly (Entry 2). In contrast, the (benzene)ruthenium catalyst gave 27 more selectively and suppressed the formation of 28 (Entry 3). Compared with the (arene)ruthenium complexes, $\mathrm{RuHCl}(\mathrm{CO})\left(\mathrm{PPh}_{3}\right)_{3}$ showed little catalytic activity, and no alkoxylation product was detected in the reaction mixture (Entry 4).

The superior performance of the (benzene)ruthenium catalyst over the ( $p$-cymene)ruthenium catalyst might be attributed to the less steric hindrance around the coordinated arenes. The intermediate of the reaction might be the hydrosilane-bound ruthenium complex via $\sigma$-coordination or oxidative addition of the Si-H bond to the ruthenium atom. Nucleophilic attack of methanol to the silicon atom having the $\mathrm{Si}-\mathrm{H}$ bond produces 27. At this step, more crowded $p$-cymene prevents methanol from attacking the central silicon atom. As a result, methanol attacks the terminal silicon atom of 26 to afford 28 via Si-Si bond cleavage.

\section{Materials and Methods}

All reactions were carried out under an argon atmosphere using standard Schlenk techniques unless otherwise noted. THF and diethyl ether were distilled from sodium benzophenone ketyl under a nitrogen atmosphere. Toluene was distilled from sodium under a nitrogen atmosphere. $\mathrm{Cl}(i-\mathrm{Pr})_{2} \mathrm{SiSi}(i-\mathrm{Pr})_{2} \mathrm{H}$ (1) [43], $\mathrm{Cl}\left(\mathrm{SiMe}_{2}\right)_{3} \mathrm{H}$ (2) [43], $\mathrm{Cl}\left(\mathrm{SiMe}_{2}\right)_{4} \mathrm{H}$ (3) [43], $\mathrm{PhMe}_{2} \mathrm{SiSi}(\mathrm{H}) \mathrm{MeSiMe}_{2} \mathrm{Ph}$ (26) [46] and $\mathrm{Et}_{3} \mathrm{SiSiEt}_{3}$ [47] were prepared according to the reported procedures. Silica gel for column 
chromatography (Kanto Chemical, silica gel 60N, spherical, neutral, particle size 100-210 $\mu \mathrm{m}$ ) was purchased and used as received. The other chemicals were purchased (Kanto Chemical, Tokyo, Japan; Kishida Chemical, Osaka, Japan; Sigma-Aldrich Japan, Tokyo, Japan; Tokyo Chemical Industry, Tokyo, Japan; Wako Pure Chemical Industries, Osaka, Japan) and used without further purification.

GC analysis was performed on a Shimadzu GC-8A gas chromatograph equipped with packed columns containing 10\% silicone SE-30 on Uniport B (GL Sciences, Tokyo, Japan) and a Shimadzu (Kyoto, Japan) C-R8A Chromatopack integrator. ${ }^{1} \mathrm{H},{ }^{13} \mathrm{C}$ and ${ }^{29} \mathrm{Si}$ NMR spectra were measured with JEOL (Akishima, Japan) JNM-ECA600, JNM-ECS400 and JNM-ECS300 spectrometers. Dodecane, tricosane or mesitylene were used as internal standards for NMR yield estimation. IR spectra were recorded on Shimadzu FTIR-8700, JASCO (Hachioji, Japan) FT/IR-4600 and hitachi (Tokyo, Japan) FTIR 270-50 spectrophotometers. Mass spectra and high-resolution mass spectra were recorded on Shimadzu GCMS-QP2010 SE and JEOL JMS-T100GCV mass spectrometers. Spectral data of all new compounds are showed in Figure S1-S113 in supplementary materials.

\subsection{Synthesis of 1,1,2,2-Tetraisopropyl-1-(2'-thienyl)disilane (4)}

A $1.64 \mathrm{M}$ solution of butyllithium in hexane $(1.5 \mathrm{~mL}, 2.5 \mathrm{mmol})$ was added dropwise to a solution of thiophene $(212 \mathrm{mg}, 2.52 \mathrm{mmol})$ in THF $(8 \mathrm{~mL})$ at $0{ }^{\circ} \mathrm{C}$, and the mixture was stirred at $0{ }^{\circ} \mathrm{C}$ for $1 \mathrm{~h}$. Compound 1 (536 mg, $2.02 \mathrm{mmol}$ ) was added, and the mixture was stirred for 2 days at room temperature. The reaction was quenched by adding $1.0 \mathrm{M}$ hydrochloric acid. The reaction mixture was extracted with hexane. The organic layer was washed with water and brine, and dried over anhydrous sodium sulfate. After evaporation of the solvents, the residue was distilled with a Kugelrohr distillation apparatus $\left(100-130{ }^{\circ} \mathrm{C} / 1 \mathrm{mmHg}\right)$ to give $4(537 \mathrm{mg}, 85 \%)$ as a yellow oil.

4. ${ }^{1} \mathrm{H}$ NMR $\left(301 \mathrm{MHz}, \mathrm{CDCl}_{3}\right): \delta 1.07(\mathrm{~d}, 6 \mathrm{H}, J=6.9 \mathrm{~Hz}), 1.10(\mathrm{~d}, 6 \mathrm{H}, J=6.9 \mathrm{~Hz}), 1.12(\mathrm{~d}, 6 \mathrm{H}, J=7.2 \mathrm{~Hz})$, $1.14(\mathrm{~d}, 6 \mathrm{H}, J=8.1 \mathrm{~Hz}), 1.16-1.31(\mathrm{~m}, 2 \mathrm{H}), 1.40(\mathrm{sept}, 2 \mathrm{H}, J=7.3 \mathrm{~Hz}), 3.73(\mathrm{t}, 1 \mathrm{H}, J=3.2 \mathrm{~Hz}), 7.20(\mathrm{dd}, 1 \mathrm{H}$, $J=4.7,3.5 \mathrm{~Hz}), 7.31(\mathrm{dd}, 1 \mathrm{H}, J=3.5,1.1 \mathrm{~Hz}), 7.61(\mathrm{dd}, 1 \mathrm{H}, J=4.7,1.1 \mathrm{~Hz}) .{ }^{13} \mathrm{C}$ NMR $\left(76 \mathrm{MHz}, \mathrm{CDCl}_{3}\right)$ : $\delta 11.7,13.7,19.3,19.5,20.8,21.6,128.0,130.6,134.8,136.2 .{ }^{29} \mathrm{Si} \mathrm{NMR}\left(60 \mathrm{MHz}, \mathrm{CDCl}_{3}\right): \delta-14.7,-9.0 . \mathrm{IR}$ (NaCl): 2940, 2860, 2070, 1460, 1210, 1000, 880, 750, $700 \mathrm{~cm}^{-1}$. MS (EI, $\left.70 \mathrm{eV}\right): \mathrm{m} / z 312\left(\mathrm{M}^{+}, 16\right)$, 269 (100), 227 (37), 197 (16), 185 (25), 155 (17), 141 (15), 127 (20). hRMS (EI): found 312.1774, calcd for $\mathrm{C}_{16} \mathrm{H}_{32} \mathrm{SSi}_{2} 312.1763$.

\subsection{Synthesis of 1,1,2,2-Tetraisopropyl-1-(4'-methoxyphenyl)disilane (5)}

A $1.53 \mathrm{M}$ solution of tert-butyllithium in pentane $(3.7 \mathrm{~mL}, 5.7 \mathrm{mmol})$ was added dropwise to a solution of 4-iodoanisole $(260 \mathrm{mg}, 1.11 \mathrm{mmol})$ in THF $(5 \mathrm{~mL})$ at $-78{ }^{\circ} \mathrm{C}$, and the mixture was stirred at $-78{ }^{\circ} \mathrm{C}$ for $1 \mathrm{~h}$. After warming to room temperature, $1(244 \mathrm{mg}, 0.921 \mathrm{mmol})$ was added, and the mixture was stirred at room temperature for $24 \mathrm{~h}$. The reaction was quenched by adding 1.0 $\mathrm{M}$ hydrochloric acid. The reaction mixture was extracted with hexane. The organic layer was washed with water and brine, and dried over anhydrous sodium sulfate. After evaporation of the solvents, the residue was distilled with a Kugelrohr distillation apparatus $\left(190-220^{\circ} \mathrm{C} / 1 \mathrm{mmHg}\right)$ to give $5(141 \mathrm{mg}, 45 \%)$ as a yellow oil.

5. ${ }^{1} \mathrm{H}$ NMR $\left(301 \mathrm{MHz}, \mathrm{CDCl}_{3}\right): \delta 1.03(\mathrm{~d}, 6 \mathrm{H}, J=7.2 \mathrm{~Hz}), 1.05(\mathrm{~d}, 6 \mathrm{H}, J=7.2 \mathrm{~Hz}), 1.08(\mathrm{~d}, 6 \mathrm{H}, J=7.2 \mathrm{~Hz})$, $1.09(\mathrm{~d}, 6 \mathrm{H}, J=7.2 \mathrm{~Hz}), 1.14-1.27(\mathrm{~m}, 2 \mathrm{H}), 1.40(\mathrm{sept}, 2 \mathrm{H}, J=7.2 \mathrm{~Hz}), 3.73(\mathrm{t}, 1 \mathrm{H}, J=3.3 \mathrm{~Hz}), 3.81(\mathrm{~s}, 3 \mathrm{H})$, $6.89(\mathrm{~d}, 2 \mathrm{H}, J=8.7 \mathrm{~Hz}), 7.45(\mathrm{~d}, 2 \mathrm{H}, J=8.7 \mathrm{~Hz}) .{ }^{13} \mathrm{C} \mathrm{NMR}\left(76 \mathrm{MHz}, \mathrm{CDCl}_{3}\right): \delta 11.7,12.7,19.2,19.4,21.0$, 21.7, 55.0, 113.4, 126.5, 137.2, 160.1. ${ }^{29} \mathrm{Si} \mathrm{NMR}\left(99 \mathrm{MHz}, \mathrm{CDCl}_{3}\right): \delta-15.0,-6.1$. IR (NaCl): 2940, 2860, 2070, 1590, 1500, 1460, 1280, 1250, 1180, $1100 \mathrm{~cm}^{-1}$. MS (EI, $\left.70 \mathrm{eV}\right): \mathrm{m} / z 336\left(\mathrm{M}^{+}, 43\right), 293$ (100), $251(48)$, 221 (77), 209 (38), 179 (43), 167 (19), 165 (16), 151 (27), 137 (14). hRMS (EI): found 336.2305, calcd for $\mathrm{C}_{19} \mathrm{H}_{36} \mathrm{OSi}_{2}$ 336.2305. 


\subsection{Representative Procedure: Synthesis of 1,1,2,2,3,3-Hexamethyl-1-(2'-thienyl)trisilane (16)}

A 1.64 M solution of butyllithium in hexane $(4.8 \mathrm{~mL}, 7.9 \mathrm{mmol})$ was added dropwise to a solution of chlorotrimethylsilane $(847 \mathrm{mg}, 7.80 \mathrm{mmol})$ in THF $(15 \mathrm{~mL})$ at $-78^{\circ} \mathrm{C}$, and the mixture was stirred at $-78^{\circ} \mathrm{C}$ for $10 \mathrm{~min}$. After warming to room temperature, magnesium turnings (392 $\left.\mathrm{mg}, 16.1 \mathrm{mmol}\right)$ and a $1.0 \mathrm{M}$ solution of DIBAL-H in toluene $(0.1 \mathrm{~mL}, 0.1 \mathrm{mmol})$ were added. After stirring for $5 \mathrm{~min}$, the mixture was cooled to $-10{ }^{\circ} \mathrm{C}$, 2-bromothiophene $(974 \mathrm{mg}, 5.97 \mathrm{mmol}$ ) was added. After stirring for $1 \mathrm{~h}, 2$ (876 mg, $4.15 \mathrm{mmol})$ was added, and the mixture was stirred at room temperature overnight. The reaction was quenched with $1.0 \mathrm{M}$ hydrochloric acid. The reaction mixture was extracted with hexane. The organic layer was washed with water and brine, and dried over anhydrous sodium sulfate. After evaporation of the solvents, the residue was separated by column chromatography over silica gel with hexane to give $\mathbf{1 6}(758 \mathrm{mg}, 71 \%)$ as a colorless oil.

16. ${ }^{1} \mathrm{H} \mathrm{NMR}\left(600 \mathrm{MHz}, \mathrm{CDCl}_{3}\right)$ : $\delta 0.09$ (d, 6H, J = $\left.4.5 \mathrm{~Hz}\right), 0.15$ (s, 6H), $0.41(\mathrm{~s}, 6 \mathrm{H}), 3.73$ (sept, 1H, $J=4.5 \mathrm{~Hz}), 7.19(\mathrm{dd}, 1 \mathrm{H}, J=4.5,3.2 \mathrm{~Hz}), 7.21(\mathrm{dd}, 1 \mathrm{H}, J=3.2,0.8 \mathrm{~Hz}), 7.59(\mathrm{dd}, 1 \mathrm{H}, J=4.5,0.8 \mathrm{~Hz})$.

${ }^{13} \mathrm{C} \mathrm{NMR}\left(151 \mathrm{MHz}, \mathrm{CDCl}_{3}\right): \delta-6.6,-5.9,-1.8,128.3,130.6,134.2,139.1 .{ }^{29} \mathrm{Si} \mathrm{NMR}\left(119 \mathrm{MHz} \mathrm{CDCl}_{3}\right)$ : $\delta-47.2,-36.3,-20.5$. IR (NaCl): 2950, 2090, 1400, 1250, 1030, 880, 830, $770 \mathrm{~cm}^{-1}$. MS (EI, $\left.70 \mathrm{eV}\right): \mathrm{m} / z$ $258\left(\mathrm{M}^{+}, 5\right), 243$ (59), 199 (21), 173 (26), 170 (23), 141 (82), 116 (52), 73 (100). hRMS (EI): found 257.0669, calcd for $\mathrm{C}_{10} \mathrm{H}_{21} \mathrm{SSi}_{3}\left(\mathrm{M}^{+}-\mathrm{h}\right) 257.0672$.

\subsection{Synthesis of 1-(5'-Hexenyl)-1,1,2,2,3,3-hexamethyltrisilane (6)}

Synthesis of 6 was carried out by the same procedure as 16 using THF (4 mL), chlorotrimethylsilane $(178 \mathrm{mg}, 1.64 \mathrm{mmol})$, a $1.64 \mathrm{M}$ solution of butyllithium in hexane $(1.14 \mathrm{~mL}$, $1.87 \mathrm{mmol})$, magnesium turnings $(95 \mathrm{mg}, 3.9 \mathrm{mmol})$, a $1.0 \mathrm{M}$ solution of DIBAL-H in toluene $(0.024 \mathrm{~mL}$, $0.024 \mathrm{mmol}), 6$-bromo-1-hexene $(234 \mathrm{mg}, 1.44 \mathrm{mmol})$ and $2(178 \mathrm{mg}, 0.844 \mathrm{mmol})$. Separation by column chromatography over silica gel with hexane gave $6(84 \mathrm{mg}, 38 \%)$ as a colorless oil.

6. ${ }^{1} \mathrm{H}$ NMR (600 MHz, $\left.\mathrm{CDCl}_{3}\right): \delta 0.06(\mathrm{~s}, 6 \mathrm{H}), 0.12(\mathrm{~s}, 6 \mathrm{H}), 0.15(\mathrm{~d}, 6 \mathrm{H}, J=4.5 \mathrm{~Hz}), 0.59-0.64(\mathrm{~m}, 2 \mathrm{H})$, 1.30-1.37 (m, 2H), 1.40-1.44 (m, 2H), 2.03-2.07 (m, 2H), 3.72 (sept, 1H, J = 4.5 Hz), 4.92-4.94 (m, 1H), 4.98-5.01 (m, 1H), 5.77-5.84 (m, 1H). $\left.{ }^{13} \mathrm{C} \mathrm{NMR} \mathrm{(151} \mathrm{MHz,} \mathrm{CDCl}_{3}\right): \delta-6.4,-5.8,-3.4,15.5,24.3,33.1$, 33.7, 114.3, 139.2. ${ }^{29} \mathrm{Si} N \mathrm{NMR}\left(119 \mathrm{MHz}_{\mathrm{CDCl}}\right): \delta-48.0,-36.1,-13.5$. IR (NaCl): 3080, 2950, 2920, 2090, 1640, 1411, 1250, 910, 880, 830, $790 \mathrm{~cm}^{-1}$. MS (EI, $\left.70 \mathrm{eV}\right): \mathrm{m} / z 258\left(\mathrm{M}^{+}, 9\right), 215$ (20), $141(64)$, 127 (18), 117 (20), 116 (19), 73 (100), 59 (51). hRMS (EI): found 258.1649, calcd for $\mathrm{C}_{12} \mathrm{H}_{30} \mathrm{Si}_{3} 258.1655$.

\subsection{Synthesis of 1,1,2,2,3,3-Hexamethyl-1-(2'-phenylethyl)trisilane (7)}

Synthesis of 7 was carried out by the same procedure as 16 using THF (20 mL), chlorotrimethylsilane $(1.01 \mathrm{~g}, 9.32 \mathrm{mmol})$, a $1.60 \mathrm{M}$ solution of butyllithium in hexane $(6.0 \mathrm{~mL}$, $9.6 \mathrm{mmol})$, magnesium turnings $(472 \mathrm{mg}, 19.4 \mathrm{mmol})$, a $1.0 \mathrm{M}$ solution of DIBAL-H in toluene $(0.12 \mathrm{~mL}$, $0.12 \mathrm{mmol})$, (2-bromoethyl)benzene $(1.30 \mathrm{~g}, 7.04 \mathrm{mmol})$ and $2(1.01 \mathrm{~g}, 4.79 \mathrm{mmol})$. Separation by column chromatography over silica gel with hexane containing 1\% triethylamine gave $7(939 \mathrm{mg}, 70 \%)$ as a colorless oil.

7. ${ }^{1} \mathrm{H}$ NMR $\left(600 \mathrm{MHz} \mathrm{CDCl}_{3}\right): \delta 0.16(\mathrm{~s}, 6 \mathrm{H}), 0.19(\mathrm{~s}, 6 \mathrm{H}), 0.21(\mathrm{~d}, 6 \mathrm{H}, J=4.5 \mathrm{~Hz}), 1.01-1.05(\mathrm{~m}, 2 \mathrm{H})$, 2.65-2.70 (m, 2H), 3.81 (sept, 1H, J = 4.5 Hz), 7.17-7.26 (m, 3H), 7.29-7.33 (m, 2H). ${ }^{13} \mathrm{C} \mathrm{NMR}(151 \mathrm{MHz}$, $\left.\mathrm{CDCl}_{3}\right): \delta-6.4,-5.8,-3.5,17.9,30.9,125.7,127.9,128.5,145.5 .{ }^{29} \mathrm{Si} \mathrm{NMR}\left(119 \mathrm{MHz}, \mathrm{CDCl}_{3}\right): \delta-47.9$, -36.2, -13.3. IR (NaCl): 3030, 2950, 2890, 2090, 1600, 1490, 1450, 1410, 1250, 880, 830, $790 \mathrm{~cm}^{-1}$. MS (EI, $70 \mathrm{eV}): \mathrm{m} / \mathrm{z} 265\left(\mathrm{M}^{+}-\mathrm{CH}_{3}, 11\right), 221$ (76), 163 (71), 135 (100), 117 (84), 116 (38), 73 (78), 59 (84). hRMS (EI): found 265.1263, calcd for $\mathrm{C}_{13} \mathrm{H}_{25} \mathrm{Si}_{3}\left(\mathrm{M}^{+}-\mathrm{CH}_{3}\right) 265.1264$.

\subsection{Synthesis of 1,1,2,2,3,3-Hexamethyl-1-(E)-styryltrisilane (8)}

Synthesis of $\mathbf{8}$ was carried out by the same procedure as $\mathbf{1 6}$ using THF (8 mL), chlorotrimethylsilane $(407 \mathrm{mg}, 3.75 \mathrm{mmol})$, a $1.64 \mathrm{M}$ solution of butyllithium in hexane $(2.3 \mathrm{~mL}$, 
$3.8 \mathrm{mmol})$, magnesium turnings ( $188 \mathrm{mg}, 7.73 \mathrm{mmol})$, a $1.0 \mathrm{M}$ solution of DIBAL-H in toluene $(0.064 \mathrm{~mL}$, $0.064 \mathrm{mmol}), \beta$-bromostyrene (527 mg, $2.88 \mathrm{mmol})$ and $2(430 \mathrm{mg}, 2.04 \mathrm{mmol})$. Separation by column chromatography over silica gel with hexane gave 8 (332 mg, 58\%) as a colorless oil.

8. ${ }^{1} \mathrm{H}$ NMR $\left(600 \mathrm{MHz}, \mathrm{CDCl}_{3}\right): \delta 0.09(\mathrm{~s}, 6 \mathrm{H}), 0.10(\mathrm{~d}, 6 \mathrm{H}, J=4.5 \mathrm{~Hz}), 0.20(\mathrm{~s}, 6 \mathrm{H}), 3.70(\mathrm{sept}, 1 \mathrm{H}$, $J=4.5 \mathrm{~Hz}), 6.45(\mathrm{~d}, 1 \mathrm{H}, J=19 \mathrm{~Hz}), 6.78(\mathrm{~d}, 1 \mathrm{H}, J=19 \mathrm{~Hz}), 7.16-7.19(\mathrm{~m}, 1 \mathrm{H}), 7.24-7.28(\mathrm{~m}, 2 \mathrm{H}), 7.33-7.38$ $(\mathrm{m}, 2 \mathrm{H}) .{ }^{13} \mathrm{C}$ NMR $\left(151 \mathrm{MHz}, \mathrm{CDCl}_{3}\right): \delta-6.5,-5.8,-3.3,126.4,128.0,128.7$ (2 peaks are overlapped.), 138.6, 143.8. ${ }^{29} \mathrm{Si} \mathrm{NMR}\left(119 \mathrm{MHz}, \mathrm{CDCl}_{3}\right): \delta-47.2,-36.1,-20.2$. IR (NaCl): 3020, 2950, 2890, 2090, 1710, 1600, 1570, 1490, 1450, 1400, 1250, 990, 880, 840, 790, $730 \mathrm{~cm}^{-1}$. MS (EI, $\left.70 \mathrm{eV}\right): \mathrm{m} / z 278\left(\mathrm{M}^{+}, 4\right)$, 219 (18), 204 (22), 161 (26), 145 (79), 135 (32), 117 (25), 116 (91), 102 (17), 73 (100), 59 (68). hRMS (EI): found 278.1334, calcd for $\mathrm{C}_{14} \mathrm{H}_{26} \mathrm{Si}_{3} 278.1342$.

\subsection{Synthesis of 1-(2-Buten-2-yl)-1,1,2,2,3,3-hexamethyltrisilane (9)}

Synthesis of 9 was carried out by the same procedure as 16 using THF (8 mL), chlorotrimethylsilane ( $407 \mathrm{mg}, 3.75 \mathrm{mmol})$, a $1.64 \mathrm{M}$ solution of butyllithium in hexane $(2.3 \mathrm{~mL}$, $3.8 \mathrm{mmol})$, magnesium turnings $(188 \mathrm{mg}, 7.73 \mathrm{mmol})$, a $1.0 \mathrm{M}$ solution of DIBAL-H in toluene $(0.048 \mathrm{~mL}$, $0.048 \mathrm{mmol}), 2$-bromo-2-butene ( $388 \mathrm{mg}, 2.87 \mathrm{mmol}$ ) and $2(420 \mathrm{mg}, 1.99 \mathrm{mmol})$. Separation by column chromatography over silica gel with hexane gave $9(248 \mathrm{mg}, 54 \%, E / Z=32 / 68)$ as a colorless oil.

(Z)-9. ${ }^{1} \mathrm{H} \mathrm{NMR}\left(600 \mathrm{MHz}, \mathrm{CDCl}_{3}\right): \delta 0.15(\mathrm{~s}, 6 \mathrm{H}), 0.15(\mathrm{~d}, 6 \mathrm{H}, J=4.8 \mathrm{~Hz}), 0.25(\mathrm{~s}, 6 \mathrm{H}), 1.67-1.71(\mathrm{~m}, 3 \mathrm{H})$, 1.74-1.76 (m, 3H), $3.74(\mathrm{sept}, 1 \mathrm{H}, J=4.2 \mathrm{~Hz}), 6.07-6.11(\mathrm{~m}, 1 \mathrm{H}) .{ }^{13} \mathrm{C} \mathrm{NMR}\left(151 \mathrm{MHz}, \mathrm{CDCl}_{3}\right): \delta-5.9$, $-5.8,-1.9,18.4,25.3,134.8,136.4 .{ }^{29} \mathrm{Si} N M R\left(119 \mathrm{MHz}, \mathrm{CDCl}_{3}\right): \delta-46.6,-35.7,-22.3$.

(E)-9. ${ }^{1} \mathrm{H}$ NMR $\left(600 \mathrm{MHz}, \mathrm{CDCl}_{3}\right): \delta 0.13(\mathrm{~d}, 6 \mathrm{H}, J=4.2 \mathrm{~Hz}), 0.15(\mathrm{~s}, 6 \mathrm{H}), 0.25(\mathrm{~s}, 6 \mathrm{H}), 1.68-1.71(\mathrm{~m}, 3 \mathrm{H})$, 1.74-1.75 (m, 3H), $3.71(\mathrm{sept}, 1 \mathrm{H}, J=4.8 \mathrm{~Hz}), 5.77-5.80(\mathrm{~m}, 1 \mathrm{H}) .{ }^{13} \mathrm{C} \mathrm{NMR}\left(151 \mathrm{MHz}, \mathrm{CDCl}_{3}\right): \delta-6.1$, $-5.9,-3.7,14.4,15.4,133.9,136.2 .{ }^{29} \mathrm{Si} N M R\left(119 \mathrm{MHz}, \mathrm{CDCl}_{3}\right): \delta-47.8,-35.7,-17.3$.

Mixture of (Z)-9 and (E)-9. IR (NaCl): 2950, 2900, 2090, 1250, 880, 830, $790 \mathrm{~cm}^{-1}$. MS (EI, $\left.70 \mathrm{eV}\right): \mathrm{m} / \mathrm{z}$ $230\left(\mathrm{M}^{+}, 7\right), 156$ (14), 141 (16), 131 (19), 117 (35), 116 (82), 97 (19), 73 (100), 59 (41). hRMS (EI): found 230.1335, calcd for $\mathrm{C}_{10} \mathrm{H}_{26} \mathrm{Si}_{3} 230.1342$.

\subsection{Synthesis of 1-(1'-Hexynyl)-1,1,2,2,3,3-hexamethyltrisilane (10)}

A $0.90 \mathrm{M}$ solution of isopropylmagnesium chloride in THF $(1.11 \mathrm{~mL}, 1.0 \mathrm{mmol})$ was added to a solution of 1-hexyne $(83 \mathrm{mg}, 1.0 \mathrm{mmol})$ in THF $(5 \mathrm{~mL})$, and the mixture was stirred at $40{ }^{\circ} \mathrm{C}$ for $1 \mathrm{~h}$. After cooling to room temperature, $2(316 \mathrm{mg}, 1.50 \mathrm{mmol})$ was added, and the mixture was stirred at room temperature for $1 \mathrm{~h}$. The reaction was quenched with $1.0 \mathrm{M}$ hydrochloric acid. The reaction mixture was extracted with hexane. The organic layer was washed with water, saturated aqueous sodium hydrogencarbonate and brine, and dried over anhydrous sodium sulfate. After evaporation of the solvents, the residue was separated by column chromatography over silica gel with hexane to give $10(123 \mathrm{mg}, 48 \%)$ as a colorless oil.

10. ${ }^{1} \mathrm{H}$ NMR $\left(600 \mathrm{MHz}, \mathrm{CDCl}_{3}\right): \delta 0.16(\mathrm{~s}, 6 \mathrm{H}), 0.18(\mathrm{~d}, 6 \mathrm{H}, J=4.5 \mathrm{~Hz}), 0.20(\mathrm{~s}, 6 \mathrm{H}), 0.90(\mathrm{t}, 3 \mathrm{H}, J=7.2 \mathrm{~Hz})$, $1.37-1.44(\mathrm{~m}, 2 \mathrm{H}), 1.46-1.51(\mathrm{~m}, 2 \mathrm{H}), 2.24(\mathrm{t}, 2 \mathrm{H}, J=6.9 \mathrm{~Hz}), 3.75(\mathrm{sept}, 1 \mathrm{H}, J=4.5 \mathrm{~Hz}) .{ }^{13} \mathrm{C}$ NMR $\left(151 \mathrm{MHz}, \mathrm{CDCl}_{3}\right): \delta-6.7,-5.8,-1.8,13.7,19.9,22.0,31.0,82.9,110.3 .{ }^{29} \mathrm{Si} \mathrm{NMR}\left(119 \mathrm{MHz}, \mathrm{CDCl}_{3}\right)$ : $\delta-46.8,-36.2,-34.9$. IR (NaCl): 2960, 2170, 2090, 1710, 1260, $800 \mathrm{~cm}^{-1}$. MS (EI, $\left.70 \mathrm{eV}\right): \mathrm{m} / \mathrm{z} 256\left(\mathrm{M}^{+}\right.$, 3), 241 (14), 197 (16), 141 (38), 139 (22), 117 (37), 116 (100), 101 (14), 83 (22), 73 (99), 59 (36). hRMS (EI): found 256.1491, calcd for $\mathrm{C}_{12} \mathrm{H}_{28} \mathrm{Si}_{3} 256.1499$.

\subsection{Synthesis of 1,1,2,2,3,3-Hexamethyl-1-(phenylethynyl)trisilane (11)}

Synthesis of $\mathbf{1 1}$ was carried out by the same procedure as $\mathbf{1 0}$ using THF ( $25 \mathrm{~mL})$, phenylacetylene $(811 \mathrm{mg}, 7.94 \mathrm{mmol})$, a $0.81 \mathrm{M}$ solution of isopropylmagnesium chloride in THF $(9.5 \mathrm{~mL}, 7.7 \mathrm{mmol})$ and $2(1.55 \mathrm{~g}, 7.36 \mathrm{mmol})$. Separation by column chromatography over silica gel with hexane containing $1 \%$ triethylamine gave $\mathbf{1 1}(1.41 \mathrm{~g}, 69 \%)$ as a colorless oil. 
11. ${ }^{1} \mathrm{H}$ NMR $\left(600 \mathrm{MHz}, \mathrm{CDCl}_{3}\right): \delta 0.22(\mathrm{~d}, 6 \mathrm{H}, J=4.5 \mathrm{~Hz}), 0.23(\mathrm{~s}, 6 \mathrm{H}), 0.31(\mathrm{~s}, 6 \mathrm{H}), 3.81$ (sept, $1 \mathrm{H}$, $J=4.5 \mathrm{~Hz}), 7.27-7.30(\mathrm{~m}, 3 \mathrm{H}), 7.44-7.46(\mathrm{~m}, 2 \mathrm{H}) .{ }^{13} \mathrm{C} \mathrm{NMR}\left(151 \mathrm{MHz}, \mathrm{CDCl}_{3}\right): \delta-6.6,-5.8,-2.1,93.3$, 107.8, 123.6, 128.3, 128.5, 132.0. $\left.{ }^{29} \mathrm{Si} \mathrm{NMR} \mathrm{(119} \mathrm{MHz,} \mathrm{CDCl}_{3}\right): \delta-46.3,-36.2,-33.7$. IR (NaCl): 2950, 2360, 2150, 1490, $1250 \mathrm{~cm}^{-1}$. MS (EI, $\left.70 \mathrm{eV}\right): \mathrm{m} / z 276\left(\mathrm{M}^{+}, 14\right), 261$ (15), 217 (63), 203 (27), 159 (64), 135 (26), 116 (71), 73 (100). hRMS (EI): found 276.1178, calcd for $\mathrm{C}_{14} \mathrm{H}_{24} \mathrm{Si}_{3} 276.1186$.

\subsection{Synthesis of 1,1,2,2,3,3-Hexamethyl-1-(trimethylsilylethynyl)trisilane (12)}

Synthesis of $\mathbf{1 2}$ was carried out by the same procedure as $\mathbf{1 0}$ using THF (5 mL), trimethylsilylacetylene $(98 \mathrm{mg}, 1.0 \mathrm{mmol})$, a $0.90 \mathrm{M}$ solution of isopropylmagnesium chloride in THF (1.11 mL, $1.0 \mathrm{mmol})$ and $2(316 \mathrm{mg}, 1.50 \mathrm{mmol})$. Separation by column chromatography over silica gel with hexane gave 12 (149 $\mathrm{mg}, 55 \%)$ as a colorless oil.

12. ${ }^{1} \mathrm{H}$ NMR $\left(600 \mathrm{MHz}, \mathrm{CDCl}_{3}\right): \delta 0.16(\mathrm{~s}, 9 \mathrm{H}), 0.17(\mathrm{~s}, 6 \mathrm{H}), 0.19(\mathrm{~d}, 6 \mathrm{H}, J=4.5 \mathrm{~Hz}), 0.22(\mathrm{~s}, 6 \mathrm{H})$, 3.77 (sept, $1 \mathrm{H}, J=4.5 \mathrm{~Hz}) .{ }^{13} \mathrm{C}$ NMR $\left(151 \mathrm{MHz}, \mathrm{CDCl}_{3}\right): \delta-6.7,-5.8,-2.2,0.1,113.0,116.9 .{ }^{29} \mathrm{Si} \mathrm{NMR}$ $\left(119 \mathrm{MHz}, \mathrm{CDCl}_{3}\right): \delta-46.6,-36.1,-34.7,-18.9$. IR (NaCl): 2960, 2090, 1260, 840, $800 \mathrm{~cm}^{-1}$. MS (EI, 70 $\mathrm{eV}): m / z 272\left(\mathrm{M}^{+}, 4\right), 257$ (12), 213 (41), 155 (18), 117 (16), 116 (100), 73 (80). hRMS (EI): found 272.1267, calcd for $\mathrm{C}_{11} \mathrm{H}_{28} \mathrm{Si}_{4} 272.1268$.

\subsection{Synthesis of 1,1,2,2,3,3-Hexamethyl-1-phenyltrisilane (13)}

Synthesis of 13 was carried out by the same procedure as 16 using THF (10 mL), chlorotrimethylsilane $(0.7 \mathrm{~mL}, 6 \mathrm{mmol})$, a $1.60 \mathrm{M}$ solution of butyllithium in hexane $(3.4 \mathrm{~mL}, 5.4 \mathrm{mmol})$, magnesium turnings $(290 \mathrm{mg}, 11.9 \mathrm{mmol})$, a $1.0 \mathrm{M}$ solution of DIBAL-H in toluene $(0.073 \mathrm{~mL}$, $0.073 \mathrm{mmol})$, bromobenzene $(0.44 \mathrm{~mL}, 4.2 \mathrm{mmol})$ and $2(609 \mathrm{mg}, 2.89 \mathrm{mmol})$. Separation by column chromatography over silica gel with hexane containing 1\% triethylamine gave $\mathbf{1 3}$ (404 $\mathrm{mg}, 55 \%$ ) as a colorless oil. The ${ }^{1} \mathrm{H}$ NMR spectrum is identical to the reported data [48].

13. ${ }^{1} \mathrm{H}$ NMR $\left(600 \mathrm{MHz}, \mathrm{CDCl}_{3}\right): \delta 0.08(\mathrm{~d}, 6 \mathrm{H}, J=4.5 \mathrm{~Hz}), 0.13(\mathrm{~s}, 6 \mathrm{H}), 0.40(\mathrm{~s}, 6 \mathrm{H}), 3.73(\mathrm{sept}, 1 \mathrm{H}$, $J=4.5 \mathrm{~Hz}), 7.33-7.36(\mathrm{~m}, 3 \mathrm{H}), 7.46-7.48(\mathrm{~m}, 2 \mathrm{H}) .{ }^{13} \mathrm{C} \mathrm{NMR}\left(151 \mathrm{MHz}, \mathrm{CDCl}_{3}\right): \delta-6.5,-5.9,-3.2,127.9$, $128.5,133.9,139.8 .{ }^{29} \mathrm{Si}$ NMR $\left(119 \mathrm{MHz}, \mathrm{CDCl}_{3}\right): \delta-47.4,-36.1,-18.0$.

\subsection{Synthesis of 1-(4'-Methoxyphenyl)-1,1,2,2,3,3-hexamethyltrisilane (14)}

Synthesis of $\mathbf{1 4}$ was carried out by the same procedure as $\mathbf{1 6}$ using THF (5 mL), chlorotrimethylsilane $(301 \mathrm{mg}, 2.77 \mathrm{mmol})$, a $1.64 \mathrm{M}$ solution of butyllithium in hexane $(1.7 \mathrm{~mL}$, $2.8 \mathrm{mmol})$, magnesium turnings ( $127 \mathrm{mg}, 5.22 \mathrm{mmol})$, a $1.0 \mathrm{M}$ solution of DIBAL-H in toluene $(0.06 \mathrm{~mL}$, $0.06 \mathrm{mmol})$, 4-bromoanisole ( $380 \mathrm{mg}, 2.03 \mathrm{mmol})$ and $2(301 \mathrm{mg}, 1.43 \mathrm{mmol})$. Separation by column chromatography over silica gel with hexane gave $14(270 \mathrm{mg}, 67 \%)$ as a colorless oil.

14. ${ }^{1} \mathrm{H}$ NMR $\left(600 \mathrm{MHz}, \mathrm{CDCl}_{3}\right): \delta 0.08(\mathrm{~d}, 6 \mathrm{H}, J=4.5 \mathrm{~Hz}), 0.11(\mathrm{~s}, 6 \mathrm{H}), 0.35(\mathrm{~s}, 6 \mathrm{H}), 3.71(\mathrm{sept}, 1 \mathrm{H}$, $J=4.5 \mathrm{~Hz}), 3.82(\mathrm{~s}, 3 \mathrm{H}), 6.90(\mathrm{~d}, 2 \mathrm{H}, J=8.7 \mathrm{~Hz}), 7.37(\mathrm{~d}, 2 \mathrm{H}, J=8.7 \mathrm{~Hz}) .{ }^{13} \mathrm{C} \mathrm{NMR}\left(151 \mathrm{MHz}, \mathrm{CDCl}_{3}\right)$ : $\delta-6.4,-5.9,-2.9,55.1,113.7,130.3,135.2,160.2 .{ }^{29} \mathrm{Si} \mathrm{NMR}\left(119 \mathrm{MHz}, \mathrm{CDCl}_{3}\right): \delta-47.5,-36.1$, -18.4. IR (NaCl): 2950, 2090, 1590, 1500, 1280, 1250, 1180, 1110, 880, 830, $780 \mathrm{~cm}^{-1}$. MS (EI, $\left.70 \mathrm{eV}\right): \mathrm{m} / \mathrm{z}$ $282\left(\mathrm{M}^{+}, 8\right), 281$ (20), 193 (36), 165 (100), 135 (16), 116 (54), 73 (29). hRMS (EI): found 281.1213, calcd for $\mathrm{C}_{13} \mathrm{H}_{25} \mathrm{OSi}_{3}\left(\mathrm{M}^{+}-\mathrm{h}\right) 281.1213$.

\subsection{Synthesis of 1-(4'-Dimethylaminophenyl)-1,1,2,2,3,3-hexamethyltrisilane (15)}

Synthesis of 15 was carried out by the same procedure as 16 using THF (5 mL), chlorotrimethylsilane $(290 \mathrm{mg}, 2.67 \mathrm{mmol})$, a $1.64 \mathrm{M}$ solution of butyllithium in hexane $(1.6 \mathrm{~mL}$, $2.6 \mathrm{mmol})$, magnesium turnings (122 mg, $5.02 \mathrm{mmol})$, a $1.0 \mathrm{M}$ solution of DIBAL-H in toluene $(0.06 \mathrm{~mL}$, $0.06 \mathrm{mmol})$, 4-bromo- $\mathrm{N}, \mathrm{N}$-dimethylaniline $(400 \mathrm{mg}, 2.00 \mathrm{mmol})$ and $2(277 \mathrm{mg}, 1.31 \mathrm{mmol})$. The crude product was separated by column chromatography over silica gel with hexane-ethyl acetate (5:1). Compound 15 (150 mg, 39\%) was obtained as a colorless oil. 
15. ${ }^{1} \mathrm{H}$ NMR $\left(600 \mathrm{MHz}, \mathrm{CDCl}_{3}\right): \delta 0.09(\mathrm{~d}, 6 \mathrm{H}, J=4.5 \mathrm{~Hz}), 0.11(\mathrm{~s}, 6 \mathrm{H}), 0.33(\mathrm{~s}, 6 \mathrm{H}), 2.95(\mathrm{~s}, 6 \mathrm{H})$, 3.71 (sept, $1 \mathrm{H}, J=4.5 \mathrm{~Hz}), 6.73(\mathrm{~d}, 2 \mathrm{H}, J=8.4 \mathrm{~Hz}), 7.32(\mathrm{~d}, 2 \mathrm{H}, J=8.4 \mathrm{~Hz}) .{ }^{13} \mathrm{C}$ NMR $\left(151 \mathrm{MHz}, \mathrm{CDCl}_{3}\right)$ : $\delta-6.4,-5.8,-2.8,40.4,112.3,124.5,135.0,150.8 .{ }^{29} \mathrm{Si} \mathrm{NMR}\left(119 \mathrm{MHz}, \mathrm{CDCl}_{3}\right): \delta-47.5,-36.0,-19.0 . \mathrm{IR}$ (NaCl): 2950, 2890, 2080, 1600, 1510, 1350, 1240, 1110, 880, 830, $790 \mathrm{~cm}^{-1}$. MS (EI, $\left.70 \mathrm{eV}\right): \mathrm{m} / z 295\left(\mathrm{M}^{+}\right.$, 15), 178 (100), 162 (9), 134 (12), 116 (9), 102 (12), 73 (9). hRMS (EI): found 294.1528, calcd for $\mathrm{C}_{14} \mathrm{H}_{28} \mathrm{NSi}_{3}$ $\left(\mathrm{M}^{+}-\mathrm{h}\right) 294.1529$.

\subsection{Synthesis of 1,1,2,2,3,3,4,4-Octamethyl-1-(2'-thienyl)tetrasilane (17)}

Synthesis of $\mathbf{1 7}$ was carried out by the same procedure as $\mathbf{1 6}$ using THF $(5 \mathrm{~mL})$, chlorotrimethylsilane $(282 \mathrm{mg}, 2.60 \mathrm{mmol})$, a $1.64 \mathrm{M}$ solution of butyllithium in hexane $(1.6 \mathrm{~mL}$, $2.6 \mathrm{mmol})$, magnesium turnings ( $127 \mathrm{mg}, 5.22 \mathrm{mmol})$, a $1.0 \mathrm{M}$ solution of DIBAL-H in toluene $(0.08 \mathrm{~mL}$, $0.08 \mathrm{mmol})$, 2-bromothiophene ( $324 \mathrm{mg}, 1.99 \mathrm{mmol})$ and $3(381 \mathrm{mg}, 1.42 \mathrm{mmol})$. Separation by column chromatography over silica gel with hexane gave 17 (191 mg, 43\%) as a colorless oil.

17. ${ }^{1} \mathrm{H}$ NMR $\left(600 \mathrm{MHz}, \mathrm{CDCl}_{3}\right): \delta 0.09(\mathrm{~s}, 6 \mathrm{H}), 0.12(\mathrm{~d}, 6 \mathrm{H}, J=4.5 \mathrm{~Hz}), 0.15(\mathrm{~s}, 6 \mathrm{H}), 0.42(\mathrm{~s}, 6 \mathrm{H})$, $3.72(\mathrm{sept}, 1 \mathrm{H}, J=4.5 \mathrm{~Hz}), 7.19(\mathrm{dd}, 1 \mathrm{H}, J=4.5,3.3 \mathrm{~Hz}), 7.20(\mathrm{dd}, 1 \mathrm{H}, J=3.0,0.6 \mathrm{~Hz}), 7.59(\mathrm{dd}, 1 \mathrm{H}, J=4.2$, $1.2 \mathrm{~Hz}) .{ }^{13} \mathrm{C}$ NMR $\left(151 \mathrm{MHz}, \mathrm{CDCl}_{3}\right): \delta-5.9,-5.81,-5.79,-1.4,128.3,130.5,134.1,139.3 .{ }^{29} \mathrm{Si} \mathrm{NMR}$ $\left(119 \mathrm{MHz}, \mathrm{CDCl}_{3}\right): \delta-44.1,-43.9,-35.9,-19.9 . \mathrm{IR}(\mathrm{NaCl}): 2950,2890,2090,1400,1250,1210,990$, 880, 830, 780, $700 \mathrm{~cm}^{-1}$. MS (EI, $\left.70 \mathrm{eV}\right): \mathrm{m} / z 316\left(\mathrm{M}^{+}, 1\right), 257$ (100), 173 (17), 167 (34), 159 (19), 141 (39), 116 (18), 73 (93), 59 (15). hRMS (EI): found 301.0753, calcd for $\mathrm{C}_{11} \mathrm{H}_{25} \mathrm{SSi}_{4}\left(\mathrm{M}^{+}-\mathrm{CH}_{3}\right) 301.0754$.

\subsection{Synthesis of 1-Methoxy-1,1,2,2,3,3-hexamethyl-3-(E)-styryltrisilane (18)}

$\left[\mathrm{RuCl}_{2}(p \text {-cymene })\right]_{2}(15 \mathrm{mg}, 0.024 \mathrm{mmol})$ was added to a solution of $8(271 \mathrm{mg}, 0.973 \mathrm{mmol})$ and methanol $(65 \mu \mathrm{L}, 1.6 \mathrm{mmol})$ in toluene $(4 \mathrm{~mL})$ at $0{ }^{\circ} \mathrm{C}$. The mixture was stirred with gradual warming to room temperature overnight. After evaporation of the solvent, the residue was distilled with a Kugelrohr distillation apparatus $\left(146-152{ }^{\circ} \mathrm{C} / 0.90-1.4 \mathrm{mmHg}\right)$ to give 18 (216 $\left.\mathrm{mg}, 72 \%\right)$ as a colorless oil.

18. ${ }^{1} \mathrm{H}$ NMR $\left(600 \mathrm{MHz}, \mathrm{CDCl}_{3}\right): \delta 0.19(\mathrm{~s}, 6 \mathrm{H}), 0.25(\mathrm{~s}, 6 \mathrm{H}), 0.28(\mathrm{~s}, 6 \mathrm{H}), 3.43(\mathrm{~s}, 3 \mathrm{H}), 6.54(\mathrm{~d}, 1 \mathrm{H}$, $J=18.9 \mathrm{~Hz}), 6.86(\mathrm{~d}, 1 \mathrm{H}, J=18.9 \mathrm{~Hz}), 7.23-7.25(\mathrm{~m}, 1 \mathrm{H}), 7.31-7.35(\mathrm{~m}, 2 \mathrm{H}), 7.42-7.45(\mathrm{~m}, 2 \mathrm{H}) .{ }^{13} \mathrm{C}$ NMR $\left(151 \mathrm{MHz}, \mathrm{CDCl}_{3}\right): \delta-6.4,-3.4,-0.1,51.4,126.4,127.9,128.58,128.64,138.6,143.8 .{ }^{29} \mathrm{Si} \mathrm{NMR}$ $\left(119 \mathrm{MHz}, \mathrm{CDCl}_{3}\right): \delta-50.2,-20.7,21.3$. IR (NaCl): 2950, 1590, 1570, 1490, 1450, 1400, 1250, 1080, 990, 830, 780, 730, $690 \mathrm{~cm}^{-1}$. MS (EI, $70 \mathrm{eV}$ ): $\mathrm{m} / z 308\left(\mathrm{M}^{+}, 10\right), 293$ (44), 219 (48), 173 (52), 145 (72), 135 (65), 133 (37), 117 (41), 116 (57), 89 (39), 73 (100), 59 (71). hRMS (FD): found 308.1449, calcd for $\mathrm{C}_{15} \mathrm{H}_{28} \mathrm{OSi}_{3}$ 308.1448 .

\subsection{Synthesis of 1-(1'-Hexynyl)-3-methoxy-1,1,2,2,3,3-hexamethyltrisilane (19)}

Synthesis of 19 was carried out by the same procedure as 18 using toluene $(4 \mathrm{~mL}), \mathbf{1 0}(264 \mathrm{mg}$, $1.03 \mathrm{mmol})$, methanol $(65 \mu \mathrm{L}, 1.6 \mathrm{mmol})$ and $\left[\mathrm{RuCl}_{2}(p \text {-cymene })\right]_{2}(15 \mathrm{mg}, 0.024 \mathrm{mmol})$. The crude product was distilled with a Kugelrohr distillation apparatus $\left(112-116^{\circ} \mathrm{C} / 1.2-1.3 \mathrm{mmHg}\right)$ to give 19 (191 $\mathrm{mg}, 65 \%$ ) as a colorless oil.

19. ${ }^{1} \mathrm{H} \mathrm{NMR}\left(600 \mathrm{MHz}, \mathrm{CDCl}_{3}\right): \delta 0.17(\mathrm{~s}, 6 \mathrm{H}), 0.20(\mathrm{~s}, 6 \mathrm{H}), 0.25(\mathrm{~s}, 6 \mathrm{H}), 0.89(\mathrm{t}, 3 \mathrm{H}, J=7.2 \mathrm{~Hz}), 1.35-1.42$ $(\mathrm{m}, 2 \mathrm{H}), 1.45-1.50(\mathrm{~m}, 2 \mathrm{H}), 2.22(\mathrm{t}, 2 \mathrm{H}, J=7.2 \mathrm{~Hz}), 3.43(\mathrm{~s}, 3 \mathrm{H}) .{ }^{13} \mathrm{C} \mathrm{NMR}\left(151 \mathrm{MHz}, \mathrm{CDCl}_{3}\right): \delta-6.6$, $-1.9,-0.1,13.7,19.9,22.0,30.9,51.4,82.9,110.2 .{ }^{29} \mathrm{Si} \mathrm{NMR}\left(119 \mathrm{MHz}, \mathrm{CDCl}_{3}\right): \delta-49.9,-35.1,21.2$. IR ( $\mathrm{NaCl}): 2960,2170,1250,1090,1040,830,780 \mathrm{~cm}^{-1}$. MS (EI, $\left.70 \mathrm{eV}\right): \mathrm{m} / z 286\left(\mathrm{M}^{+}, 1\right), 257$ (19), 229 (16), 133 (20), 117 (35), 116 (54), 89 (41), 73 (100), 59 (61). hRMS (FD): found 286.1612, calcd for $\mathrm{C}_{13} \mathrm{H}_{30} \mathrm{OSi}_{3}$ 286.1604 . 


\subsection{Synthesis of 1-Methoxy-1,1,2,2,3,3-hexamethyl-3-(phenylethynyl)trisilane (20)}

Synthesis of $\mathbf{2 0}$ was carried out by the same procedure as $\mathbf{1 8}$ using toluene $(4 \mathrm{~mL}), \mathbf{1 1}(280 \mathrm{mg}$, $1.01 \mathrm{mmol})$, methanol $(65 \mu \mathrm{L}, 1.6 \mathrm{mmol})$ and $\left[\mathrm{RuCl}_{2}(p \text {-cymene })\right]_{2}(15 \mathrm{mg}, 0.024 \mathrm{mmol})$. The crude product was distilled with a Kugelrohr distillation apparatus $\left(134-135{ }^{\circ} \mathrm{C} / 0.90-0.93 \mathrm{mmHg}\right)$ to give 20 (147 $\mathrm{mg}, 47 \%)$ as a colorless oil.

20. ${ }^{1} \mathrm{H}$ NMR $\left(600 \mathrm{MHz}, \mathrm{CDCl}_{3}\right): \delta 0.25(\mathrm{~s}, 6 \mathrm{H}), 0.30(\mathrm{~s}, 6 \mathrm{H}), 0.33(\mathrm{~s}, 6 \mathrm{H}), 3.46(\mathrm{~s}, 3 \mathrm{H}), 7.29-7.30(\mathrm{~m}, 3 \mathrm{H})$, 7.43-7.45 (m, 2H). ${ }^{13} \mathrm{C}$ NMR $\left(151 \mathrm{MHz}, \mathrm{CDCl}_{3}\right): \delta-6.5,-2.1,0.0,51.5,93.3,107.8,123.5,128.3,128.5$, 132.0. ${ }^{29} \mathrm{Si}$ NMR (119 MHz, $\left.\mathrm{CDCl}_{3}\right): \delta-49.5,-33.9,21.0$. IR (NaCl): 2970, 2940, 2900, 2150, 1490, 1250, 1080, 840, 780, $690 \mathrm{~cm}^{-1}$. MS (EI, $\left.70 \mathrm{eV}\right): \mathrm{m} / z 306\left(\mathrm{M}^{+}, 7\right), 305$ (23), 193 (41), 159 (37), 116 (34), 89 (30), 73 (100), 59 (64). hRMS (FD): found 306.1293, calcd for $\mathrm{C}_{15} \mathrm{H}_{26} \mathrm{OSi}_{3} 306.1291$.

\subsection{Synthesis of 1-Methoxy-1,1,2,2,3,3-hexamethyl-3-phenyltrisilane (21)}

Synthesis of $\mathbf{2 1}$ was carried out by the same procedure as $\mathbf{1 8}$ using toluene $(4 \mathrm{~mL}), \mathbf{1 3}(258 \mathrm{mg}$, $1.02 \mathrm{mmol})$, methanol $(60 \mu \mathrm{L}, 1.5 \mathrm{mmol})$ and $\left[\mathrm{RuCl}_{2}(p \text {-cymene })\right]_{2}(15 \mathrm{mg}, 0.024 \mathrm{mmol})$. The crude product was distilled with a Kugelrohr distillation apparatus $\left(153-157^{\circ} \mathrm{C} / 11 \mathrm{mmHg}\right)$ to give 21 ( $251 \mathrm{mg}, 87 \%$ ) as a colorless oil. The NMR data are identical to the reported data [48].

21. ${ }^{1} \mathrm{H}$ NMR $\left(600 \mathrm{MHz}, \mathrm{CDCl}_{3}\right): \delta 0.156(\mathrm{~s}, 6 \mathrm{H}), 0.160(\mathrm{~s}, 6 \mathrm{H}), 0.42(\mathrm{~s}, 6 \mathrm{H}), 3.35(\mathrm{~s}, 3 \mathrm{H}), 7.32-7.37$ $(\mathrm{m}, 3 \mathrm{H}), 7.46-7.49(\mathrm{~m}, 2 \mathrm{H}) .{ }^{13} \mathrm{C}$ NMR $\left(151 \mathrm{MHz}, \mathrm{CDCl}_{3}\right): \delta-6.3,-3.2,-0.2,51.3,127.9,128.5,133.9$, 139.6. ${ }^{29} \mathrm{Si} \mathrm{NMR}\left(119 \mathrm{MHz}, \mathrm{CDCl}_{3}\right): \delta-50.2,-18.4,21.3$. IR (NaCl): 2950, 2890, 1250, 1080, 830, 780, 730, $700 \mathrm{~cm}^{-1}$. MS (EI, $\left.70 \mathrm{eV}\right): \mathrm{m} / z 282\left(\mathrm{M}^{+}, 2\right), 267$ (38), 193 (14), 135 (54), 116 (100), 89 (15), 73 (23), 59 (17). hRMS (FD): found 282.1300, calcd for $\mathrm{C}_{13} \mathrm{H}_{26} \mathrm{OSi}_{3} 282.1291$.

\subsection{Synthesis of 1,2-Dichloro-1,1,2,2-tetraethyldisilane}

Freshly distilled acetyl chloride $(14.0 \mathrm{~mL}, 197 \mathrm{mmol})$ was added dropwise to the mixture of aluminum chloride $(26.04 \mathrm{~g}, 195 \mathrm{mmol})$ and hexaethyldisilane $(21.8 \mathrm{~mL}, 79.5 \mathrm{mmol})$. The flask was immersed in a water bath to prevent raising the reaction temperature too much. After stirring for $3 \mathrm{~h}$, the reaction mixture was distilled under reduced pressure (bp $100-131{ }^{\circ} \mathrm{C} / 9 \mathrm{mmHg}$ ) to give 1,2-dichloro-1,1,2,2-tetraethyldisilane (16.47 $\mathrm{g}$, 85\%) as a colorless oil. The ${ }^{1} \mathrm{H}$ NMR spectrum is identical to the reported data [14].

\subsection{Synthesis of 1,1,2,2-Tetraethyl-1-phenyldisilane (22)}

Phenylmagnesium bromide was prepared from magnesium turnings $(2.17 \mathrm{~g}, 89.4 \mathrm{mmol})$, bromobenzene (11.72 g, $74.6 \mathrm{mmol})$ and diethyl ether $(42 \mathrm{~mL})$. The Grignard reagent was added dropwise to a solution of 1,2-dichloro-1,1,2,2-tetraethyldisilane (16.47 g, $67.7 \mathrm{mmol})$ in diethyl ether $(30 \mathrm{~mL})$ at $0{ }^{\circ} \mathrm{C}$. The mixture was stirred at room temperature for $12 \mathrm{~h}$. After filtration of the reaction mixture, the filtrate was evaporated to give a crude product of 1-chloro-1,1,2,2-tetraethyl-2phenyldisilane (19.29 g).

A solution of 1-chloro-1,1,2,2-tetraethyl-2-phenyldisilane in diethyl ether (60 mL) was added dropwise to a mixture of lithium aluminum hydride $(1.21 \mathrm{~g}, 32.0 \mathrm{mmol})$ and diethyl ether $(100 \mathrm{~mL})$ at $0{ }^{\circ} \mathrm{C}$. The mixture was stirred with gradual warming to room temperature overnight. The reaction was quenched with 1.0 M hydrochloric acid. The reaction mixture was extracted with hexane. The organic layer was washed with brine and dried over anhydrous sodium sulfate. After evaporation of the solvents, the residue was distilled under reduced pressure according to a normal distillation procedure (bp $129-131^{\circ} \mathrm{C} / 5 \mathrm{mmHg}$ ) to give $22(12.30 \mathrm{~g}, 73 \%$ in two steps) as a colorless oil.

22. ${ }^{1} \mathrm{H}$ NMR $\left(600 \mathrm{MHz}, \mathrm{CDCl}_{3}\right): \delta 0.69-0.76(\mathrm{~m}, 4 \mathrm{H}), 0.95-1.02(\mathrm{~m}, 10 \mathrm{H}), 1.03-1.06(\mathrm{~m}, 6 \mathrm{H}), 3.67$ (quin, $1 \mathrm{H}, J=3.8 \mathrm{~Hz}), 7.33-7.36(\mathrm{~m}, 3 \mathrm{H}), 7.48-7.50(\mathrm{~m}, 2 \mathrm{H}) .{ }^{13} \mathrm{C} \mathrm{NMR}\left(151 \mathrm{MHz}, \mathrm{CDCl}_{3}\right): \delta 2.2,4.4,8.4,10.2$, 127.9, 128.6, 134.6, 137.6. ${ }^{29} \mathrm{Si}$ NMR (119 MHz, $\left.\mathrm{CDCl}_{3}\right): \delta-26.4,-13.1 . \mathrm{IR}(\mathrm{NaCl}): 3070,2950,2910$, 
2870, 2080, 1460, 1430, 1230, 1100, 1010, 970, 800, 770, $700 \mathrm{~cm}^{-1}$. MS (EI, $\left.70 \mathrm{eV}\right): \mathrm{m} / z 250\left(\mathrm{M}^{+}, 13\right)$, 163 (61), 135 (100), 107 (55). hRMS (FD): found 250.1574, calcd for $\mathrm{C}_{14} \mathrm{H}_{26} \mathrm{Si}_{2} 250.1573$.

\subsection{Synthesis of 1,1,2,2-Tetraethyl-1-methoxy-2-phenyldisilane (23)}

$\left[\mathrm{RuCl}_{2}(p \text {-cymene })\right]_{2}(16 \mathrm{mg}, 0.026 \mathrm{mmol})$ was added to a solution of $22(260 \mathrm{mg}, 1.04 \mathrm{mmol})$ and methanol $(338 \mathrm{mg}, 10.5 \mathrm{mmol})$ in toluene $(4 \mathrm{~mL})$ at $0{ }^{\circ} \mathrm{C}$. The mixture was stirred with gradual warming to room temperature for 1 day. After evaporation of the solvent, the residue was distilled with a Kugelrohr distillation apparatus $\left(81-117^{\circ} \mathrm{C} / 0.5 \mathrm{mmHg}\right)$ to give $23(241 \mathrm{mg}, 83 \%)$ as a colorless oil.

23. ${ }^{1} \mathrm{H}$ NMR (600 MHz, $\left.\mathrm{CDCl}_{3}\right): \delta 0.70-0.81(\mathrm{~m}, 4 \mathrm{H}), 0.95-1.02(\mathrm{~m}, 10 \mathrm{H}), 1.06-1.10(\mathrm{~m}, 6 \mathrm{H}), 3.41(\mathrm{~s}, 3 \mathrm{H})$, 7.31-7.36 (m, 3H), 7.51-7.53 (m, 2H). $\left.{ }^{13} \mathrm{C} \mathrm{NMR} \mathrm{(151} \mathrm{MHz,} \mathrm{CDCl}_{3}\right): \delta 4.4,7.0,7.3,8.3,51.7,127.9,128.5$, 134.8, 137.7. ${ }^{29} \mathrm{Si} \mathrm{NMR}\left(119 \mathrm{MHz}, \mathrm{CDCl}_{3}\right): \delta-17.3,19.2$. IR (NaCl): 3070, 2960, 2880, 1460, 1430, 1090, 1010, $700 \mathrm{~cm}^{-1}$. MS (EI, $\left.70 \mathrm{eV}\right): m / z 280\left(\mathrm{M}^{+}, 40\right), 279$ (100), 265 (21), 251 (20), 237 (40), 223 (27), 209 (28), 195 (23), 135 (39), 117 (25), 107 (61), 89 (35), 59 (21). hRMS (FD): found 280.1670, calcd for $\mathrm{C}_{15} \mathrm{H}_{28} \mathrm{OSi}_{2}$ 280.1678 .

\subsection{Synthesis of 1-Ethoxy-1,1,2,2-tetraethyl-2-phenyldisilane (24)}

Synthesis of 24 was carried out by the almost same procedure as 23 using toluene $(4 \mathrm{~mL})$, $22(253 \mathrm{mg}, 1.01 \mathrm{mmol})$, ethanol (476 mg, $10.3 \mathrm{mmol})$ and $\left[\mathrm{RuCl}_{2}(p \text {-cymene) }]_{2}(15 \mathrm{mg}, 0.024 \mathrm{mmol})\right.$. The mixture was stirred at $50{ }^{\circ} \mathrm{C}$ for 1 day. The solvent was evaporated, and the residue was distilled with a Kugelrohr distillation apparatus $\left(95-110^{\circ} \mathrm{C} / 0.5 \mathrm{mmHg}\right)$ to give $24(135 \mathrm{mg}, 45 \%)$ as a colorless oil.

24. ${ }^{1} \mathrm{H}$ NMR $\left(600 \mathrm{MHz}, \mathrm{CDCl}_{3}\right): \delta 0.69-0.80(\mathrm{~m}, 4 \mathrm{H}), 0.94-1.01(\mathrm{~m}, 10 \mathrm{H}), 1.05-1.08(\mathrm{~m}, 6 \mathrm{H}), 1.14(\mathrm{t}, 3 \mathrm{H}$, $J=7.0 \mathrm{~Hz}), 3.61(\mathrm{q}, 2 \mathrm{H}, J=7.0 \mathrm{~Hz}), 7.32-7.35(\mathrm{~m}, 3 \mathrm{H}), 7.49-7.53(\mathrm{~m}, 2 \mathrm{H}) .{ }^{13} \mathrm{C} \mathrm{NMR}\left(151 \mathrm{MHz}, \mathrm{CDCl}_{3}\right)$ : $\delta 4.4,7.1,7.6,8.3,18.8,59.5,127.9,128.5,134.8,137.9 .{ }^{29} \mathrm{Si} \mathrm{NMR}\left(119 \mathrm{MHz}, \mathrm{CDCl}_{3}\right): \delta-17.4,16.5$. IR (NaCl): 2960, 2880, 1460, 1430, 1100, 1080, 1000, $700 \mathrm{~cm}^{-1}$. MS (EI, $\left.70 \mathrm{eV}\right): \mathrm{m} / z 294\left(\mathrm{M}^{+}, 2\right), 293(4)$, 265 (21), 237 (37), 209 (33), 135 (36), 131 (47), 107 (100), 105 (39), 103 (63), 87 (19), 75 (35), 59 (23). hRMS (FD): found 294.1849, calcd for $\mathrm{C}_{16} \mathrm{H}_{30} \mathrm{OSi}_{2} 294.1835$.

\subsection{Synthesis of 1,1,2,2-Tetraethyl-1-isopropoxy-2-phenyldisilane (25)}

$\left[\mathrm{RuCl}_{2}(p \text {-cymene })\right]_{2}(17 \mathrm{mg}, 0.028 \mathrm{mmol})$ was added to a solution of $22(265 \mathrm{mg}, 1.06 \mathrm{mmol})$ and 2-propanol $(675 \mathrm{mg}, 11.2 \mathrm{mmol})$ in toluene $(4 \mathrm{~mL})$ at $0{ }^{\circ} \mathrm{C}$. The mixture was stirred with gradual warming to room temperature for $12 \mathrm{~h}$. Additional 2-propanol $(5.84 \mathrm{~g}, 97.1 \mathrm{mmol})$ was added, and the mixture was heated at $50{ }^{\circ} \mathrm{C}$ for 1 day. After cooling to room temperature, the solvent and excess 2-propanol were evaporated, and the residue was distilled with a Kugelrohr distillation apparatus (91-111 $\left.{ }^{\circ} \mathrm{C} / 0.5 \mathrm{mmHg}\right)$ to give $25(188 \mathrm{mg}, 58 \%)$ as a colorless oil.

25. ${ }^{1} \mathrm{H}$ NMR $\left(600 \mathrm{MHz}, \mathrm{CDCl}_{3}\right): \delta 0.71-0.75(\mathrm{~m}, 4 \mathrm{H}), 0.93-1.00(\mathrm{~m}, 10 \mathrm{H}), 1.04-1.07(\mathrm{~m}, 6 \mathrm{H}), 1.09(\mathrm{~d}, 6 \mathrm{H}$, $J=6.0 \mathrm{~Hz}), 3.90(\mathrm{sept}, 1 \mathrm{H}, J=6.0 \mathrm{~Hz}), 7.31-7.34(\mathrm{~m}, 3 \mathrm{H}), 7.51-7.53(\mathrm{~m}, 2 \mathrm{H}) .{ }^{13} \mathrm{C} \mathrm{NMR}\left(151 \mathrm{MHz}, \mathrm{CDCl}_{3}\right)$ : $\delta 4.4,7.3,8.2,8.4,26.1,66.0,127.8,128.4,134.8,138.0 .{ }^{29} \mathrm{Si} \mathrm{NMR}\left(119 \mathrm{MHz}, \mathrm{CDCl}_{3}\right): \delta-17.5,14.1$. IR (NaCl): 2960, 2880, 1460, 1430, 1380, 1120, 1020, $700 \mathrm{~cm}^{-1}$. MS (EI, $\left.70 \mathrm{eV}\right): \mathrm{m} / z 308\left(\mathrm{M}^{+}, 0.2\right), 265$ (73), 237 (84), 221 (17), 209 (54), 193 (25), 181 (14), 165 (15), 145 (41), 135 (41), 107 (100), 105 (35), 103 (79), 75 (75). hRMS (FD): found 308.1997, calcd for $\mathrm{C}_{17} \mathrm{H}_{32} \mathrm{OSi}_{2} 308.1991$.

\subsection{Synthesis of 2-Methoxy-1,1,2,3,3-pentamethyl-1,3-diphenyltrisilane (27)}

$\left[\mathrm{RuCl}_{2} \text { (benzene) }\right]_{2}(13 \mathrm{mg}, 0.026 \mathrm{mmol})$ was added to a solution of $26(317 \mathrm{mg}, 1.01 \mathrm{mmol})$ and methanol $(328 \mathrm{mg}, 10.2 \mathrm{mmol})$ in toluene $(4 \mathrm{~mL})$ at $0{ }^{\circ} \mathrm{C}$. The mixture was stirred with gradual warming to room temperature for 1 day and at $50{ }^{\circ} \mathrm{C}$ for 5 days. After cooling to room temperature, the solvent was evaporated, and the residue was distilled with a Kugelrohr distillation apparatus $\left(128-149^{\circ} \mathrm{C} / 0.5 \mathrm{mmHg}\right)$ to give $27(100 \mathrm{mg}, 29 \%)$ as a colorless oil. 
27. ${ }^{1} \mathrm{H}$ NMR $\left(600 \mathrm{MHz} \mathrm{CDCl}_{3}\right): \delta 0.32(\mathrm{~s}, 6 \mathrm{H}), 0.34(\mathrm{~s}, 6 \mathrm{H}), 0.37(\mathrm{~s}, 3 \mathrm{H}), 3.32(\mathrm{~s}, 3 \mathrm{H}), 7.32-7.33(\mathrm{~m}, 6 \mathrm{H})$, 7.42-7.43 (m, 4H). ${ }^{13} \mathrm{C} \mathrm{NMR}\left(151 \mathrm{MHz}, \mathrm{CDCl}_{3}\right): \delta-3.8,-2.90,-2.87,53.2,127.9,128.7,134.1,139.1$. ${ }^{29} \mathrm{Si} \mathrm{NMR}\left(119 \mathrm{MHz}, \mathrm{CDCl}_{3}\right): \delta-21.8,12.6$. IR (NaCl): 3070, 2950, 1430, 1250, 1070, 770, 730, $700 \mathrm{~cm}^{-1}$. MS (EI, $70 \mathrm{eV}): m / z 344\left(\mathrm{M}^{+}\right.$, 5), 329 (25), 251 (14), 209 (17), 197 (10), 193 (13), 179 (33), 178 (54), 163 (24), 135 (100), 122 (11), 117 (11), 107 (11), 105 (12), 59 (17). hRMS (FD): found 344.1460, calcd for $\mathrm{C}_{18} \mathrm{H}_{28} \mathrm{OSi}_{3}$ 344.1447.

\section{Conclusions}

We found that $\alpha$-chloro- $\omega$-hydrooligosilanes, synthesized by the titanium-catalyzed monoreduction of $\alpha, \omega$-dichlorooligosilanes, are good precursors for the synthesis of unsymmetrically substituted oligosilanes. Each functional group, the chlorosilane and hydrosilane moieties, is independently substituted by the reactions with organolithium or Grignard reagents and the ruthenium-catalyzed alkoxylations. In both steps, little or no cleavage of the Si-Si bond occurred. Further studies on these synthetic reactions are now in progress.

Supplementary Materials: The following are available online at http:/ /www.mdpi.com/2304-6740/6/3/99/s1, Figures S1-S113: spectral data of all new compounds.

Author Contributions: Conceptualization, K.-i.K.; Experiments, Y.A., Y.N., M.I. and K.K.; Project Administration, S.K.; Preparation of the manuscript, K.-i.K. and S.K.

Funding: This work was supported in part by Grants-in-Aid for Scientific Research (Nos. 23550044 and 26410036) from the Japan Society for the Promotion of Science and the Element Innovation Project of Gunma University, Japan.

Acknowledgments: Some of the spectral data of the new compounds were measured in the Instrumental Analysis Division, Equipment Management Center, Creative Research Institution, hokkaido University, Japan.

Conflicts of Interest: The authors declare no conflict of interest.

\section{References}

1. Miller, R.D.; Michl, J. Polysilane high polymers. Chem. Rev. 1989, 89, 1359-1410. [CrossRef]

2. Beckmann, J. Oligosilanes. In Comprehensive Organometallic Chemistry III; Crabtree, R.H., Mingos, D.M.P., Eds.; Elsevier: Oxford, UK, 2007; Volume 3, pp. 409-512.

3. Marschner, C. Oligosilanes. In Functional Molecular Silicon Compounds I: Regular Oxidation States; Scheschkewitz, D., Ed.; Springer: Cham, Switzerland, 2014; pp. 163-228.

4. Mignani, G.; Krämer, A.; Puccetti, G.; Ledoux, I.; Soula, G.; Zyss, J.; Meyrueix, R. A new class of silicon compounds with interesting nonlinear optical effects. Organometallics 1990, 9, 2640-2643. [CrossRef]

5. Mignani, G.; Krämer, A.; Puccetti, G.; Ledoux, I.; Zyss, J.; Soula, G. Effect of a weak donor on the intramolecular charge transfer of molecules containing two neighboring silicon atoms. Organometallics 1991, 10, 3656-3659. [CrossRef]

6. Mignani, G.; Barzoukas, M.; Zyss, J.; Soula, G.; Balegroune, F.; Grandjean, D.; Josse, D. Improved transparency-efficiency trade-off in a new class of nonlinear organosilicon compounds. Organometallics 1991, 10, 3660-3668. [CrossRef]

7. Tsuji, H.; Sasaki, M.; Shibano, Y.; Toganoh, M.; Kataoka, T.; Araki, Y.; Tamao, K.; Ito, O. Photoinduced electron transfer of dialkynyldisilane-linked zinc porphyrin-[60]fullerene dyad. Bull. Chem. Soc. Jpn. 2006, 79, 1338-1346. [CrossRef]

8. Shibano, Y.; Sasaki, M.; Tsuji, H.; Araki, Y.; Ito, O.; Tamao, K. Conformation effect of oligosilane linker on photoinduced electron transfer of tetrasilane-linked zinc porphyrin-[60]fullerene dyads. J. Organomet. Chem. 2007, 692, 356-367. [CrossRef]

9. Sasaki, M.; Shibano, Y.; Tsuji, H.; Araki, Y.; Tamao, K.; Ito, O. Oligosilane chain-length dependence of electron transfer of zinc porphyrin-oligosilane-fullerene molecules. J. Phys. Chem. A 2007, 111, 2973-2979. [CrossRef] [PubMed]

10. Hiratsuka, H.; horiuchi, H.; Takanoha, Y.; Matsumoto, H.; Yoshihara, T.; Okutsu, T.; Negishi, K.; Kyushin, S.; Matsumoto, H. Excited-state property of 1-(4-cyanophenyl)-2-(4-methoxyphenyl)-1,1,2,2-tetramethyldisilane. Chem. Lett. 2007, 36, 1168-1169. [CrossRef] 
11. Iwamoto, T.; Tsushima, D.; Kwon, E.; Ishida, S.; Isobe, H. Persilastaffanes: Design, synthesis, structure, and conjugation between silicon cages. Angew. Chem., Int. Ed. 2012, 51, 2340-2344. [CrossRef] [PubMed]

12. Surampudi, S.; Yeh, M.-L.; Siegler, M.A.; hardigree, J.F.M.; Kasl, T.A.; Katz, H.E.; Klausen, R.S. Increased carrier mobility in end-functionalized oligosilanes. Chem. Sci. 2015, 6, 1905-1909. [CrossRef] [PubMed]

13. Shimada, M.; Yamanoi, Y.; Matsushita, T.; Kondo, T.; Nishibori, E.; hatakeyama, A.; Sugimoto, K.; Nishihara, H. Optical properties of disilane-bridged donor-acceptor architectures: Strong effect of substituents on fluorescence and nonlinear optical properties. J. Am. Chem. Soc. 2015, 137, 1024-1027. [CrossRef] [PubMed]

14. Kunai, A.; Kawakami, T.; Toyoda, E.; Ishikawa, M. highly selective synthesis of chlorosilanes from hydrosilanes. Organometallics 1992, 11, 2708-2711. [CrossRef]

15. Kunai, A.; Ochi, T.; Iwata, A.; Ohshita, J. Synthesis of bromohydrosilanes: Reactions of hydrosilanes with $\mathrm{CuBr}_{2}$ in the presence of CuI. Chem. Lett. 2001, 1228-1229. [CrossRef]

16. Kunai, A.; Ohshita, J. Selective synthesis of halosilanes from hydrosilanes and utilization for organic synthesis. J. Organomet. Chem. 2003, 686, 3-15. [CrossRef]

17. Harrison, D.J.; McDonald, R.; Rosenberg, L. Borane-catalyzed hydrosilylation of thiobenzophenone: A new route to silicon-sulfur bond formation. Organometallics 2005, 24, 1398-1400. [CrossRef]

18. Kato, N.; Tamura, Y.; Kashiwabara, T.; Sanji, T.; Tanaka, M. $\mathrm{AlCl}_{3}$-catalyzed hydrosilylation of alkynes with hydropolysilanes. Organometallics 2010, 29, 5274-5282. [CrossRef]

19. Oestreich, M.; hermeke, J.; Mohr, J. A unified survey of $\mathrm{Si}-\mathrm{H}$ and h-H bond activation catalysed by electron-deficient boranes. Chem. Soc. Rev. 2015, 44, 2202-2220. [CrossRef] [PubMed]

20. Chatgilialoglu, C. Organosilanes in Radical Chemistry; Wiley: Chichester, UK, 2004.

21. Chatgilialoglu, $\mathrm{C}$. $\left(\mathrm{Me}_{3} \mathrm{Si}\right)_{3} \mathrm{SiH}$ : Twenty years after its discovery as a radical-based reducing agent. Chem. Eur. J. 2008, 14, 2310-2320. [CrossRef] [PubMed]

22. Urenovitch, J.V.; West, R. Pentamethyldisilane and 1,1,2,2-tetramethyldisilane and their addition to olefins. J. Organomet. Chem. 1965, 3, 138-145. [CrossRef]

23. Sakurai, H.; Kishida, T.; hosomi, A.; Kumada, M. Decomposition of some free radical initiators in hexamethyldisilane. J. Organomet. Chem. 1967, 8, 65-68. [CrossRef]

24. Hsiao, Y.-L.; Waymouth, R.M. Free-radical hydrosilylation of poly(phenylsilane): Synthesis of functional polysilanes. J. Am. Chem. Soc. 1994, 116, 9779-9780. [CrossRef]

25. Ojima, I. The hydrosilylation reaction. In The Chemistry of Organic Silicon Compounds; Patai, S., Rappoport, Z., Eds.; Wiley: Chichester, UK, 1989; pp. 1479-1526.

26. Ojima, I.; Li, Z.; Zhu, J. Recent advances in the hydrosilylation and related reactions. In The Chemistry of Organic Silicon Compounds; Rappoport, Z., Apeloig, Y., Eds.; Wiley: Chichester, UK, 1998; Volume 2, pp. 1687-1792.

27. Marciniec, B.; Maciejewski, H.; Pietraszuk, C.; Pawluć, P. Hydrosilylation: A Comprehensive Review on Recent Advances; Marciniec, B., Ed.; Springer: Berlin, Germany, 2009.

28. Lukevics, E.; Dzintara, M. The alcoholysis of hydrosilanes. J. Organomet. Chem. 1985, 295, 265-315. [CrossRef]

29. Corey, J.Y. Dehydrogenative coupling reactions of hydrosilanes. In Advances in Silicon Chemistry; Larson, G.L., Ed.; JAI Press: Greenwich, CT, USA, 1991; Volume 1, pp. 327-387.

30. Gauvin, F.; harrod, J.F.; Woo, H.G. Catalytic dehydrocoupling: A general strategy for the formation of element-element bonds. Adv. Organomet. Chem. 1998, 42, 363-405.

31. Reichl, J.A.; Berry, D.H. Recent progress in transition metal-catalyzed reactions of silicon, germanium, and tin. Adv. Organomet. Chem. 1999, 43, 197-265.

32. Murata, M.; Suzuki, K.; Watanabe, S.; Masuda, Y. Synthesis of arylsilanes via palladium(0)-catalyzed silylation of aryl halides with hydrosilane. J. Org. Chem. 1997, 62, 8569-8571. [CrossRef] [PubMed]

33. Tsukada, N.; hartwig, J.F. Intermolecular and intramolecular, platinum-catalyzed, acceptorless dehydrogenative coupling of hydrosilanes with aryl and aliphatic methyl C-H bonds. J. Am. Chem. Soc. 2005, 127, 5022-5023. [CrossRef] [PubMed]

34. Yamanoi, Y. Palladium-catalyzed silylations of hydrosilanes with aryl halides using bulky alkyl phosphine. J. Org. Chem. 2005, 70, 9607-9609. [CrossRef] [PubMed]

35. Murata, M.; Yamasaki, H.; Ueta, T.; Nagata, M.; Ishikura, M.; Watanabe, S.; Masuda, Y. Synthesis of aryltriethoxysilanes via rhodium(I)-catalyzed cross-coupling of aryl electrophiles with triethoxysilane. Tetrahedron 2007, 63, 4087-4094. [CrossRef] 
36. Yamanoi, Y.; Nishihara, H. Direct and selective arylation of tertiary silanes with rhodium catalyst. J. Org. Chem. 2008, 73, 6671-6678. [CrossRef] [PubMed]

37. Horn, K.A. Regio- and stereochemical aspects of the palladium-catalyzed reactions of silanes. Chem. Rev. 1995, 95, 1317-1350. [CrossRef]

38. Sharma, H.K.; Pannell, K.H. Activation of the Si-Si bond by transition metal complexes. Chem. Rev. 1995, 95, 1351-1374. [CrossRef]

39. Suginome, M.; Ito, Y. Transition-metal-catalyzed additions of silicon-silicon and silicon-heteroatom bonds to unsaturated organic molecules. Chem. Rev. 2000, 100, 3221-3256. [CrossRef] [PubMed]

40. Beletskaya, I.; Moberg, C. Element-element additions to unsaturated carbon-carbon bonds catalyzed by transition metal complexes. Chem. Rev. 2006, 106, 2320-2354. [CrossRef] [PubMed]

41. Lesbani, A.; Kondo, H.; Sato, J.; Yamanoi, Y.; Nishihara, H. Facile synthesis of hypersilylated aromatic compounds by palladium-mediated arylation reaction. Chem. Commun. 2010, 46, 7784-7786. [CrossRef] [PubMed]

42. Inubushi, H.; hattori, Y.; Yamanoi, Y.; Nishihara, H. Structures and optical properties of tris(trimethylsilyl)silylated oligothiophene derivatives. J. Org. Chem. 2014, 79, 2974-2979. [CrossRef] [PubMed]

43. Kanno, K.; Niwayama, Y.; Kyushin, S. Selective catalytic monoreduction of dichlorooligosilanes with Grignard reagents. Tetrahedron Lett. 2013, 54, 6940-6943. [CrossRef]

44. Kanno, K.; Aikawa, Y.; Kyushin, S. Ruthenium-catalyzed alkoxylation of a hydrodisilane without Si-Si bond cleavage. Tetrahedron Lett. 2017, 58, 9-12. [CrossRef]

45. Piller, F.M.; Metzger, A.; Schade, M.A.; haag, B.A.; Gavryushin, A.; Knochel, P. Preparation of polyfunctional arylmagnesium, arylzinc, and benzylic zinc reagents by using magnesium in the presence of LiCl. Chem. Eur. J. 2009, 15, 7192-7202. [CrossRef] [PubMed]

46. Russell, A.G.; Guveli, T.; Kariuki, B.M.; Snaith, J.S. Synthesis and characterisation of two new binaphthyl trisilanes. J. Organomet. Chem. 2009, 694, 137-141. [CrossRef]

47. Ahmed, M.A.K.; Wragg, D.S.; Nilsen, O.; Fjellvåg, H. Synthesis and properties of ethyl, propyl, and butyl hexa-alkyldisilanes and tetrakis(tri-alkylsilyl)silanes. Z. Anorg. Allg. Chem. 2014, 640, 2956-2961. [CrossRef]

48. Hoffmann, F.; Wagler, J.; Roewer, G. Selective synthesis of functional alkynylmono- and -trisilanes. Eur. J. Inorg. Chem. 2010, 1133-1142. [CrossRef] 